CERN-TH/2001-366 IFUP-TH/2001-41 IFIC/01-69 $\quad$ FTUV-01-1217 RM3-TH/2001-17

\title{
Old and new physics interpretations of the NuTeV anomaly
}

\author{
S. Davidson ${ }^{a, b}$, S. Forte ${ }^{c}$ P. Gambino ${ }^{d}$, N. Rius ${ }^{a}$, A. Strumia $^{d 2}$ \\ (a) Depto. de Fisica Teórica and IFIC, Universidad de Valencia-CSIC, Valencia, Spain \\ (b) IPPP, University of Durham, Durham DH1 3LE,UK \\ (c) INFN, Sezione di Roma III, Via della Vasca Navale, I-00146, Roma, Italy \\ (d) Theoretical Physics Division, CERN, CH-1211 Genève 23, Suisse
}

\begin{abstract}
We discuss whether the $\mathrm{NuTeV}$ anomaly can be explained, compatibly with all other data, by QCD effects (maybe, if the strange sea is asymmetric, or there is a tiny violation of isospin), new physics in propagators or couplings of the vector bosons (not really), loops of supersymmetric particles (no), dimension six operators (yes, for one specific $\mathrm{SU}(2)_{L}$-invariant operator), leptoquarks (not in a minimal way), extra $\mathrm{U}(1)$ gauge bosons (maybe: an unmixed $Z^{\prime}$ coupled to $B-3 L_{\mu}$ also increases the muon $g-2$ by about $10^{-9}$ and gives a 'burst' to cosmic rays above the GZK cutoff).
\end{abstract}

\section{Introduction}

The NuTeV collaboration [四] has recently reported a $\sim 3 \sigma$ anomaly in the NC/CC ratio of deepinelastic $\nu_{\mu}$-nucleon scattering. The effective $\nu_{\mu}$ coupling to left-handed quarks is found to be about $1 \%$ lower than the best fit SM prediction.

As in the case of other apparent anomalies (e.g. $\epsilon^{\prime} / \epsilon$ [2, 3], the muon $g-2$ [4, 旬, 6], atomic parity violation [7, 8], and another puzzling $\mathrm{NuTeV}$ result concerning dimuon events [9], to cite only the most recent cases) one should first worry about theoretical uncertainties, mainly due to QCD, before speculating on possible new physics. After reviewing in section 2 the SM prediction for the NuTeV observables, in section 3 we look for SM effects and/or uncertainties which could alleviate the anomaly. In particular, we investigate the possible effect of next-to-leading order QCD corrections and consider the uncertainties related to parton distribution functions (PDFs). We notice that a small asymmetry between strange and antistrange in the quark sea of the nucleon, suggested by $\nu \mathcal{N}$ deep inelastic data [10], could be responsible for a significant fraction of the observed anomaly. We also study the effect a very small violation of isospin symmetry can have on the $\mathrm{NuTeV}$ result.

\footnotetext{
${ }^{1}$ On leave from INFN, Sezione di Torino, Italy

${ }^{2}$ On leave from dipartimento di Fisica dell'Università di Pisa and INFN.
} 


\begin{tabular}{|lrcc|}
\hline $\mathrm{SM}$ fermion & $\mathrm{U}(1)_{Y}$ & $\mathrm{SU}(2)_{L}$ & $\mathrm{SU}(3)_{\mathrm{c}}$ \\
\hline$U^{c}=u_{R}^{c}$ & $-\frac{2}{3}$ & 1 & $\overline{3}$ \\
$D^{c}=d_{R}^{c}$ & $\frac{1}{3}$ & 1 & $\overline{3}$ \\
$E^{c}=e_{R}^{c}$ & 1 & 1 & 1 \\
$L=\left(\nu_{L}, e_{L}\right)$ & $-\frac{1}{2}$ & 2 & 1 \\
$Q=\left(u_{L}, d_{L}\right)$ & $\frac{1}{6}$ & 2 & 3 \\
\hline
\end{tabular}

\begin{tabular}{|c|cc|}
\hline$Z$ couplings & $g_{L}$ & $g_{R}$ \\
\hline$\nu_{e}, \nu_{\mu}, \nu_{\tau}$ & $\frac{1}{2}$ & 0 \\
$e, \mu, \tau$ & $-\frac{1}{2}+s_{\mathrm{W}}^{2}$ & $s_{\mathrm{W}}^{2}$ \\
$u, c, t$ & $\frac{1}{2}-\frac{2}{3} s_{\mathrm{W}}^{2}$ & $-\frac{2}{3} s_{\mathrm{W}}^{2}$ \\
$d, s, b$ & $-\frac{1}{2}+\frac{1}{3} s_{\mathrm{W}}^{2}$ & $\frac{1}{3} s_{\mathrm{W}}^{2}$ \\
\hline
\end{tabular}

Table 1: The SM fermions and their $Z$ couplings.

Having looked at the possible SM explanations, and keeping in mind that large statistical fluctuations cannot be excluded, we then speculate on the sort of physics beyond the SM that could be responsible for the $\mathrm{NuTeV}$ anomaly. We make a broad review of the main mechanisms through which new physics may affect the quantities measured at $\mathrm{NuTeV}$ and test them quantitatively, taking into account all the constraints coming from other data.

We take the point of view that interesting models should be able to explain a significant fraction of the anomaly. According to this criterion, we consider new physics that only affects the propagators (section (1) or gauge interactions (section 5) of the SM vector bosons, looking at the constraints imposed on them by a global fit to the electroweak precision observables. Many models can generate a small fraction of the observed discrepancy (see e.g. [1]), but it is more difficult to explain a significant fraction of the anomaly. In section 6 we consider the case of the minimal supersymmetric SM (MSSM) and look at possible MSSM quantum effects. In section 7 we turn to lepton-lepton-quark-quark effective vertices, focusing on the most generic set of dimension 6 operators. We find that very few of them can fit the $\mathrm{NuTeV}$ anomaly (in particular, only one $\mathrm{SU}(2)_{L}$-invariant operator). In section 8 and 9 we study how these dimension six operators could be generated by exchange of leptoquarks or of extra U(1) gauge bosons. Finally, we summarize our findings in section 10.

\section{The SM prediction}

\section{Tree level}

In order to establish the notation and to present the physics in a simple approximation, it is useful to recall the tree-level SM prediction for neutrino-nucleon deep inelastic scattering. The $\nu_{\mu}$-quark effective Lagrangian predicted by the SM at tree level is

$$
\mathscr{L}_{\text {eff }}=-2 \sqrt{2} G_{F}\left(\left[\bar{\nu}_{\mu} \gamma_{\alpha} \mu_{L}\right]\left[\bar{d}_{L} \gamma^{\alpha} u_{L}\right]+\text { h.c. }\right)-2 \sqrt{2} G_{F} \sum_{A, q} g_{A q}\left[\bar{\nu}_{\mu} \gamma_{\alpha} \nu_{\mu}\right]\left[\bar{q}_{A} \gamma^{\alpha} q_{A}\right]
$$

where $A=\{L, R\}, q=\{u, d, s, \ldots\}$ and the $Z$ couplings $g_{A q}$ are given in table 1 in terms of the weak mixing angle $s_{\mathrm{W}} \equiv \sin \theta_{\mathrm{W}}$.

It is convenient to define the ratios of neutral-current (NC) to charged-current (CC) deep-inelastic neutrino-nucleon scattering total cross-sections $R_{\nu}, R_{\bar{\nu}}$. Including only first generation quarks, for an isoscalar target, and to leading order, these are given by

$$
\begin{aligned}
& R_{\nu} \equiv \frac{\sigma(\nu \mathcal{N} \rightarrow \nu X)}{\sigma(\nu \mathcal{N} \rightarrow \mu X)}=\frac{\left(3 g_{L}^{2}+g_{R}^{2}\right) q+\left(3 g_{R}^{2}+g_{L}^{2}\right) \bar{q}}{3 q+\bar{q}}=g_{L}^{2}+r g_{R}^{2} \\
& R_{\bar{\nu}} \equiv \frac{\sigma(\bar{\nu} \mathcal{N} \rightarrow \bar{\nu} X)}{\sigma(\bar{\nu} \mathcal{N} \rightarrow \bar{\mu} X)}=\frac{\left(3 g_{R}^{2}+g_{L}^{2}\right) q+\left(3 g_{L}^{2}+g_{R}^{2}\right) \bar{q}}{q+3 \bar{q}}=g_{L}^{2}+\frac{1}{r} g_{R}^{2}
\end{aligned}
$$


where $q$ and $\bar{q}$ denote the second moments of quark or antiquark distributions and correspond to the fraction of the nucleon momentum carried by quarks and antiquarks, respectively. For an isoscalar target, $q=(u+d) / 2$, and we have defined

$$
r \equiv \frac{\sigma(\bar{\nu} \mathcal{N} \rightarrow \bar{\mu} X)}{\sigma(\nu \mathcal{N} \rightarrow \mu X)}=\frac{3 \bar{q}+q}{3 q+\bar{q}}
$$

and

$$
g_{L}^{2} \equiv g_{L u}^{2}+g_{L d}^{2}=\frac{1}{2}-\sin ^{2} \theta_{\mathrm{W}}+\frac{5}{9} \sin ^{4} \theta_{\mathrm{W}}, \quad g_{R}^{2} \equiv g_{R u}^{2}+g_{R d}^{2}=\frac{5}{9} \sin ^{4} \theta_{\mathrm{W}} .
$$

The observables $R_{\nu}^{\exp }$ and $R_{\bar{\nu}}^{\exp }$ measured at $\mathrm{NuTeV}$ differ from the expressions given in eq. (2). On the theoretical side this is due to contributions from second-generation quarks, and because of QCD and electroweak corrections. On the experimental side, this is because total cross-sections can only be determined up to experimental cuts and uncertainties, such as those related to the spectrum of the neutrino beam, the contamination of the $\nu_{\mu}$ beam by electron neutrinos, and the efficiency of $\mathrm{NC} / \mathrm{CC}$ discrimination. Once all these effects are taken into account, the $\mathrm{NuTeV}$ data can be viewed as a measurement of the ratios between the $\mathrm{CC}$ and the NC squared neutrino effective couplings. The values quoted in [1] are

$$
g_{L}^{2}=0.3005 \pm 0.0014 \quad \text { and } \quad g_{R}^{2}=0.0310 \pm 0.0011,
$$

where errors include both statistical and systematic uncertainties.

The difference of the effective couplings $g_{L}^{2}-g_{R}^{2}$ ('Paschos-Wolfenstein ratio' [12]) is subject to smaller theoretical and systematic uncertainties than the individual couplings. Indeed, using eq. (2) we get

$$
R_{\mathrm{PW}} \equiv \frac{R_{\nu}-r R_{\bar{\nu}}}{1-r}=\frac{\sigma(\nu \mathcal{N} \rightarrow \nu X)-\sigma(\bar{\nu} \mathcal{N} \rightarrow \bar{\nu} X)}{\sigma(\nu \mathcal{N} \rightarrow \ell X)-\sigma(\bar{\nu} \mathcal{N} \rightarrow \bar{\ell} X)}=g_{L}^{2}-g_{R}^{2}=\frac{1}{2}-\sin ^{2} \theta_{\mathrm{W}}
$$

which is seen to be independent of $q$ and $\bar{q}$, and therefore of the information on the partonic structure of the nucleon. Also, $R_{\mathrm{PW}}$ is expected to be less sensitive to the various corrections discussed above.

\section{Electroweak corrections and the SM fit}

The tree level SM predictions for $g_{L}$ and $g_{R}$ get modified by electroweak radiative corrections. These corrections depend on the precise definition of the weak mixing angle, and we therefore adopt the on-shell definition [15] and define $s_{\mathrm{W}}^{2} \equiv 1-M_{W}^{2} / M_{Z}^{2}$. One then obtains the following expressions for $g_{L, R}^{2} 16$

$$
g_{L}^{2}=\rho^{2}\left(\frac{1}{2}-s_{\mathrm{W}}^{2} k+\frac{5}{9} s_{\mathrm{W}}^{4} k^{2}\right), \quad g_{R}^{2}=\frac{5}{9} \rho^{2} s_{\mathrm{W}}^{4} k^{2},
$$

where, also including the most important QCD and electroweak higher order effects [17]

$$
\begin{aligned}
& \rho \approx 1.0086+0.0001\left(M_{t} / \mathrm{GeV}-175\right)-0.0006 \ln \left(m_{h} / 100 \mathrm{GeV}\right) \\
& k \approx 1.0349+0.0004\left(M_{t} / \mathrm{GeV}-175\right)-0.0029 \ln \left(m_{h} / 100 \mathrm{GeV}\right)
\end{aligned}
$$

for values of the top mass, $M_{t}$, and of Higgs mass, $m_{h}$, not far from 175 and $100 \mathrm{GeV}$. Additional very small non-factorizable terms are induced by electroweak box diagrams.[?

\footnotetext{
${ }^{2}$ QED radiative corrections depend sensitively on the experimental setup and are taken into account in the NuTeV analysis. The charged current amplitude receives a small residual renormalization which depends on the implementation of QED corrections and is therefore neglected in our analysis.
} 


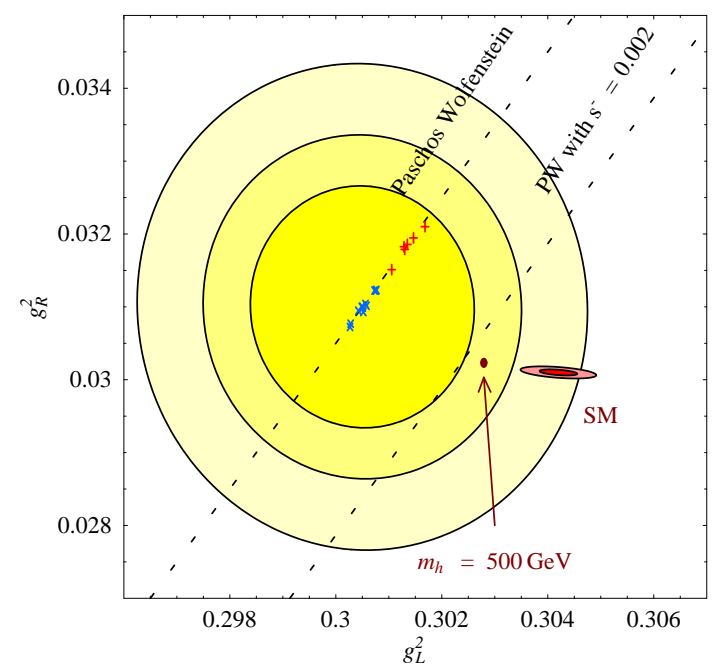

Figure 1: The SM prediction for $\left(g_{L}^{2}, g_{R}^{2}\right)$ at 68,99\% CL and the NuTeV determination, at 68,90,99\% CL. The crosses show how the $N u T e V$ central value moves along the $P W$ line using different sets of parton distribution functions that assume $s=\bar{s}$. If $s>\bar{s}$ the $P W$ line is shifted towards the SM prediction.

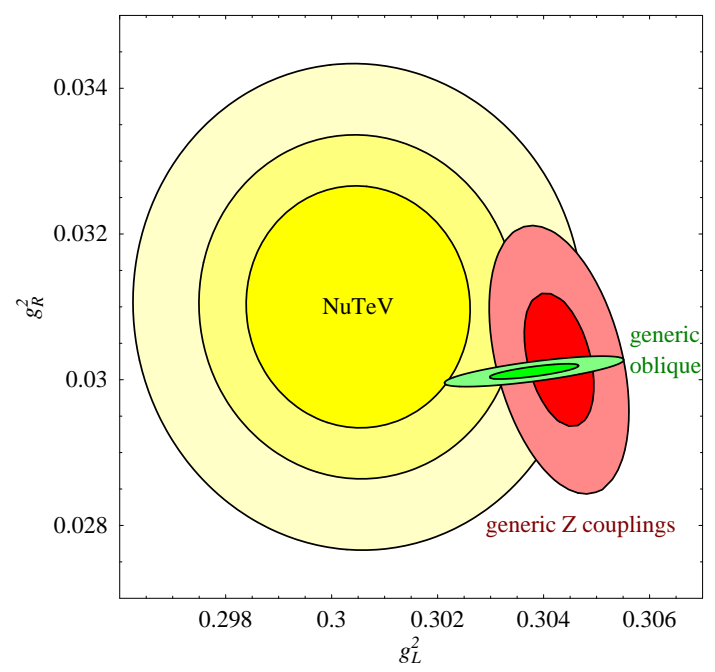

Figure 2: The NuTeV measurement is compared with the $68,99 \%$ CL ranges of $\left(g_{L}^{2}, g_{R}^{2}\right)$ allowed by precision data in classes of extensions of the $S M$ that allow generic oblique corrections (almost horizontal small green ellipses) or flavouruniversal and $\mathrm{SU}(2)_{L}$-invariant corrections of the $Z$ couplings (vertical medium red ellipses).

We stress that the SM value of $s_{\mathrm{W}}$ depends on $M_{t}$ and $m_{h}$, unless only the direct measurements of $M_{W}$ and $M_{Z}$ are used to compute it. In particular, for fixed values of $M_{t}$ and $m_{h}$, the $W$ mass can be very precisely determined from $G_{F}, \alpha\left(M_{Z}\right)$, and $M_{Z}$. To very good approximation one has (see e.g. [18])

$$
M_{W}=80.387-0.058 \ln \left(m_{h} / 100 \mathrm{GeV}\right)-0.008 \ln ^{2}\left(m_{h} / 100 \mathrm{GeV}\right)+0.0062\left(M_{t} / \mathrm{GeV}-175\right) .
$$

Without including the NuTeV results, the latest SM global fit of precision observables gives $M_{t}=$ $176.1 \pm 4.3 \mathrm{GeV}, m_{h}=87_{-34}^{+51} \mathrm{GeV}$, from which one obtains $M_{W}=80.400 \pm 0.019 \mathrm{GeV}$ [19], and therefore $s_{\mathrm{W}}^{2}=0.2226 \pm 0.0004$. The values of $g_{L}^{2}$ and $g_{R}^{2}$ corresponding to the best fit are 0.3042 and 0.0301, respectively. The small red ellipses in fig. 1 show the SM predictions for $g_{L}^{2}$ and $g_{R}^{2}$ at $68 \%$ and $99 \% \mathrm{CL}$, while the bigger yellow ellipses are the NuTeV data, at $68 \% 90 \%$ and $99 \%$ CL. While $g_{R}^{2}$ is in agreement with the SM, $g_{L}^{2}$ shows a discrepancy of about $2.5 \sigma$.

Here we have adopted the on-shell definition of $s_{\mathrm{W}}$ because it is well known that with this choice the electroweak radiative corrections to $g_{L}^{2}$ cancel to a large extent. In fact, at first order in $\delta \rho \equiv \rho-1$ and $\delta k \equiv k-1, g_{L}^{2}$ gets shifted by $\delta g_{L}^{2} \approx(2 \delta \rho-0.551 \delta k) g_{L}^{2}$ and the leading quadratic dependence on $M_{t}$ is the same for $\delta \rho$ and $\delta k s_{\mathrm{W}}^{2} / c_{\mathrm{W}}^{2}$. Therefore, the top mass sensitivity of $g_{L}^{2}$ is very limited when this effective coupling is expressed in terms of $s_{\mathrm{W}}^{2}=1-M_{W}^{2} / M_{Z}^{2}$. As leading higher order electroweak corrections are usually related to the high value of the top mass [17], higher order corrections cannot have any relevant impact on the discrepancy between the SM and $\mathrm{NuTeV}$.

Within the SM, one can extract a value of $s_{\mathrm{W}}^{2}$ from the $\mathrm{NuTeV}$ data. This is performed by the 
$\mathrm{NuTeV}$ collaboration using a fit to $s_{\mathrm{W}}^{2}$ and the effective charm mass that is used to describe the charm threshold. This fit is different from the one that gives $g_{L, R}^{2}$. The result is $m_{c}^{\text {eff }}=1.32 \pm 0.11 \mathrm{GeV}$ and

$$
\begin{aligned}
s_{\mathrm{W}}^{2}= & 0.2276 \pm 0.0013 \text { (stat.) } \pm 0.0006 \text { (syst.) } \pm 0.0006 \text { (th.) } \\
& -0.00003\left(M_{t} / \mathrm{GeV}-175\right)+0.00032 \ln \left(m_{h} / 100 \mathrm{GeV}\right) .
\end{aligned}
$$

Here the systematic uncertainty includes all the sources of experimental systematics, such as those mentioned above, and it is estimated by means of a Monte Carlo simulation of the experiment. The theoretical uncertainty is almost entirely given by QCD corrections, to be discussed in section 3 . The total uncertainty above is about $3 / 4$ of that in the preliminary result from $\mathrm{NuTeV} s_{\mathrm{W}}^{2}=0.2255 \pm$ 0.0021 [13] and about $1 / 2$ of that in the CCFR result $s_{\mathrm{W}}^{2}=0.2236 \pm 0.0035$ [14].

Alternatively, by equating eq. (7) to the $\mathrm{NuTeV}$ results for $g_{L, R}^{2}$, we find

$$
s_{\mathrm{W}}^{2}(\mathrm{NuTeV})=0.2272 \pm 0.0017 \pm 0.0001(\text { top }) \pm 0.0002 \text { (Higgs) } .
$$

The central value and the errors have been computed using the best global fit for $M_{t}$ and $m_{h}$. Eq. (10) has a slightly larger error but is very close to eq. (9). An additional difference between the two determinations is that eq. (10) is based on a up-to-date treatment of higher order effects. Notice that the $\mathrm{NuTeV}$ error is much larger than in the global fit given above, from which eq. (9) differs by about $3 \sigma$ and eq. (10) by about $2.6 \sigma$. The NuTeV result for $s_{\mathrm{W}}^{2}$ can also be re-expressed in terms of $M_{W}$. If we then compare with $M_{W}=80.451 \pm 0.033 \mathrm{GeV}$ from direct measurements at LEP and Tevatron, the discrepancy is even higher: more than $3 \sigma$ in both cases. The inclusion of the NuTeV data in a global fit shifts the preferred $m_{h}$ value very slightly, but worsens the fit significantly $\left(\chi^{2}=30\right.$ for 14 degrees of freedom).

Even without including $\mathrm{NuTeV}$ data, the global SM fit has a somewhat low goodness-of-fit, $8 \%$ if naïvely estimated with a global Pearson $\chi^{2}$ test. The quality of the fit becomes considerably worse if only the most precise data are retained [20]. Indeed, among the most precise observables, the leptonic asymmetries measured at LEP and SLD and $M_{W}$ point to an extremely light Higgs, well below the direct exclusion bound $m_{h}>115 \mathrm{GeV}$, while the forward-backward hadronic asymmetries measured at LEP prefer a very heavy Higgs (for a detailed discussion, see [20, 21]). The effective leptonic couplings measured by the hadronic asymmetries differ by more then $3 \sigma$ from those measured by purely leptonic asymmetries. Therefore, the discrepancy between $\mathrm{NuTeV}$ and the other data depends also on how this other discrepancy is treated. For instance, a fit which excludes the hadronic asymmetries has a satisfactory goodness-of-fit, but $m_{h}=40 \mathrm{GeV}$ as best fit value. In this case, the SM central values for $g_{L, R}^{2}$ are 0.3046 and 0.0299 , and differ even more from the NuTeV measurements. On the other hand, even a very heavy Higgs would not resolve the anomaly: to explain completely the NuTeV result $m_{h}$ should be as heavy as $3 \mathrm{TeV}$, deep in the non-perturbative regime. The preference of the $\mathrm{NuTeV}$ result for a heavy Higgs is illustrated in fig. 11 where we display the point corresponding to the SM predictions with $m_{h}=500 \mathrm{GeV}$ and $m_{t}=175 \mathrm{GeV}$. This is suggestive that, as will be more clearly seen in the following, the central value of $\mathrm{NuTeV}$ cannot be explained by radiative corrections.

\section{QCD corrections}

Most of the quoted theoretical error on the $\mathrm{NuTeV}$ determination of $s_{\mathrm{W}}^{2}$ is due to QCD effects. Yet, this uncertainty does not include some of the assumptions on which the Paschos-Wolfenstein relation, eq. (6), is based. Hence, one may ask: first, whether some source of violation of the PaschosWolfenstein relation which has not been included in the experimental analysis can explain the observed 
discrepancy, and second, whether some of the theoretical uncertainties might actually be larger than estimated in [1].

A full next-to-leading order (NLO) treatment of neutrino deep-inelastic scattering is possible, since all the relevant coefficient functions are long known [22]. If no assumption on the parton content of the target is made, including NLO corrections, the Paschos-Wolfenstein ratio eq. (6) becomes

$$
\begin{aligned}
R_{\mathrm{PW}}= & g_{L}^{2}-g_{R}^{2}+\frac{\left(u^{-}-d^{-}\right)+\left(c^{-}-s^{-}\right)}{\mathcal{Q}^{-}}\left\{\left[\frac{3}{2}\left(g_{L u}^{2}-g_{R u}^{2}\right)+\frac{1}{2}\left(g_{L d}^{2}-g_{R d}^{2}\right)\right]+\right. \\
& \left.+\frac{\alpha_{s}}{2 \pi}\left(g_{L}^{2}-g_{R}^{2}\right)\left(\frac{1}{4} \delta C^{1}-\delta C^{3}\right)\right\}+O\left(\mathcal{Q}^{-}\right)^{-2}
\end{aligned}
$$

The various quantities which enter eq. (11) are defined as follows: $\alpha_{s}$ is the strong coupling; $\delta C^{1} \equiv$ $C^{1}-C^{2}, \delta C^{3} \equiv C^{3}-C^{2} ; C^{i}$ is the the second moment of the next-to-leading contributions to the quark coefficient functions for structure function $F^{i} ; q^{-} \equiv q-\bar{q} ; \mathcal{Q}^{-} \equiv\left(u^{-}+d^{-}\right) / 2 ; u$, $d$, and so on are second moments of the corresponding quark and antiquark distributions. We have expanded the result in powers of $1 / \mathcal{Q}^{-}$, since we are interested in the case of targets where the dominant parton is the isoscalar $\mathcal{Q}^{-}=\left(u^{-}+d^{-}\right) / 2$. Equation (11) shows the well-known fact that the PaschosWolfenstein relation is corrected if either the target has an isotriplet component (i.e. $u \neq d$ ) or sea quark contributions have a $C$-odd component (i.e. $s^{-} \neq 0$ or $c^{-} \neq 0$ ). Furthermore, NLO corrections only affect these isotriplet or $C$-odd terms.

Let us now consider these corrections in turn. Momentum fractions are scale dependent; in the energy range of the $\mathrm{NuTeV}$ experiment $\mathcal{Q}^{-} \approx 0.18$ [23], with better than $10 \%$ accuracy, so that $\left[\frac{3}{2}\left(g_{L u}^{2}-g_{R u}^{2}\right)+\frac{1}{2}\left(g_{L d}^{2}-g_{R d}^{2}\right)\right] / \mathcal{Q}^{-} \approx 1.3$. Hence, a value $\left(u^{-}-d^{-}\right)+\left(c^{-}-s^{-}\right) \approx-0.0038$ is required to shift the value of $s_{\mathrm{W}}^{2}$ by an amount equal to the difference between the $\mathrm{NuTeV}$ value central eq. (9) and the global SM fit.

The $\mathrm{NuTeV}$ experiment uses an iron target, which has an excess of neutrons over protons of about $6 \%$. This violation of isoscalarity is however known to good accuracy, it is included in the NuTeV analysis, and it gives a negligible contribution to the overall error. A further violation of isoscalarity could be due to the fact that isospin symmetry is violated by the parton distributions of the nucleon, i.e. $u^{p} \neq d^{n}$ and $u^{n} \neq d^{p}$. This effect is considered by NuTeV [1], but not included in their analysis. Indeed, isospin in QCD is only violated by terms of order $\left(m_{u}-m_{d}\right) / \Lambda$, and thus isospin violation is expected to be smaller than $1 \%$ or so at the $\mathrm{NuTeV}$ scale (where the scale dependence is rather weak) 24]. However, if one were to conservatively estimate the associated uncertainty by assuming isospin violation of the valence distribution to be at most $1 \%$ (i.e. $\left(u^{-}-d^{-}\right) / \mathcal{Q}^{-} \leq 0.01$ ), this would lead to a theoretical uncertainty on $s_{\mathrm{W}}^{2}$ of order $\Delta s_{\mathrm{W}}^{2}=0.002$. This is a more than threefold increase in theoretical uncertainty, which would rather reduce the significance of the $\mathrm{NuTeV}$ anomaly.

A $C$-odd second moment of heavier flavours, $s^{-} \neq 0$ or $c^{-} \neq 0$ is not forbidden by any symmetry of QCD, which only imposes that the first moments of all heavy flavours must vanish. Neither of these effects has been considered by $\mathrm{NuTeV}$. A nonzero value of $c^{-}$appears very unlikely since the perturbatively generated charm component has $c^{-}=0$ identically for all moments, and even assuming that there is an 'intrinsic' charm component (i.e. $c \neq 0$ below charm threshold due to nonperturbative effects) it is expected to have vanishing $c^{-}$[25] for all moments. On the contrary, because the relevant threshold is in the nonperturbative region, the strange component is determined by infrared dynamics and there is no reason why $s^{-}=0$. In fact, explicit model calculations [26] suggest $s^{-} \approx 0.002$. Whereas such a $C$-odd strange component was at first ruled out by CCFR dimuon data [27], a

\footnotetext{
${ }^{3}$ Deep-inelastic charm production events are know as dimuon events because their experimental signature is a pair
} 
subsequent global fit to all available neutrino data found evidence in favor of a strange component of this magnitude and sign [10], and showed that it does not necessarily contradict the direct CCFR measurement. More recent measurements [28] confirm the CCFR results in a wider kinematic region; however, the quantitative impact of these data on a global fit is unclear. Even though it is not included in current parton sets, a small asymmetry $s^{-} \approx 0.002$ seems compatible with all the present experimental information [29]. Assuming $s^{-} \approx 0.002$ as suggested by [10], the value of $s_{\mathrm{W}}^{2}$ measured by $\mathrm{NuTeV}$ is lowered by about $\delta s_{\mathrm{W}}^{2}=0.0026$. The corresponding shift of the PW line is displayed in fig. 1. This reduces the discrepancy between $\mathrm{NuTeV}$ and the $\mathrm{SM}$ to the level of about one and a half standard deviations (taking the $\mathrm{NuTeV}$ error at face value), thus eliminating the anomaly.

Since NLO corrections in eq. (11) only affect the $C$-odd or isospin-odd terms, they are in practice a sub-subleading effect. Numerically, $\delta C^{1}-4 \delta C^{3}=16 / 9$ so NLO effects will merely correct a possible isotriplet or $C$-odd contribution by making it larger by a few percent. Therefore, a purely leading-order analysis of $R_{\mathrm{PW}}$ is entirely adequate, and neglect of NLO corrections should not contribute significantly to either the central value of $s_{\mathrm{W}}^{2}$ extracted from $R_{\mathrm{PW}}$, nor to the error on it. It is important to realize, however, that this is not the case when considering the individual ratios $R_{\nu}$ and $R_{\bar{\nu}}$. Indeed, NLO corrections affect the leading-order expressions by terms proportional to the dominant quark component $\mathcal{Q}^{-}$, and also by terms proportional to the gluon distribution, which carries about $50 \%$ of the nucleon's momentum. Therefore, one expects NLO corrections to $R_{\nu}$ and $R_{\bar{\nu}}$ to be of the same size as NLO corrections to typical observables at this scale, i.e. around $10 \%$. The impact of this on the values of $g_{L}^{2}$ and $g_{R}^{2}$, however, is difficult to assess: the NuTeV analysis makes use of a parton set which has been self-consistently determined fitting leading-order expressions to neutrino data, so part of the NLO correction is in effect included in the parton distributions. A reliable determination of $g_{L}^{2}$ and $g_{R}^{2}$ could only be obtained if the whole $\mathrm{NuTeV}$ analysis were consistently upgraded to NLO. As things stand, one should be aware that the $\mathrm{NuTeV}$ determination of $g_{L}^{2}$ and $g_{R}^{2}$, eq. (5), is affected by a theoretical uncertainty related to NLO which has not been taken into account and which may well be non-negligible. This uncertainty is however correlated between $g_{L}^{2}$ and $g_{R}^{2}$, and it cancels when evaluating the difference $g_{L}^{2}-g_{R}^{2}$.

On top of explicit violations of the Paschos-Wolfenstein relation, other sources of uncertainty are due to the fact that the experiment of course does not quite measure total cross-sections. Therefore, some of the dependence on the structure of the nucleon which cancels in ideal observables such as $R_{\nu}$ or $R_{\mathrm{PW}}$ remains in actual experimental observables. In order to estimate these uncertainties, we have developed a simple Monte Carlo which simulates the $\mathrm{NuTeV}$ experimental set-up. The Monte Carlo calculates integrated cross sections with cuts typical of a $\nu \mathcal{N}$ experiment, by using leadingorder expressions. Because the Monte Carlo is not fitted self-consistently to the experimental raw data, it is unlikely to give an accurate description of actual data. However, it can be used to assess the uncertainties involved in various aspects of the analysis.

We have therefore studied the variation of the result for $R_{\mathrm{PW}}$ as several theoretical assumptions are varied, none of which affects the ideal observable $R_{\mathrm{PW}}$ but all of which affect the experimental results. First, we have considered the dependence on parton distributions. Although the error on parton distributions cannot really be assessed at present, it is unlikely to be much larger than the difference between leading-order and NLO parton sets. We can study this variation by comparing the CTEQL and CTEQM parton sets [30]. We also compare results to those of the MRST99 set [23]. We find extremely small variation for $R_{\mathrm{PW}}$ and small variations even for the extraction of $g_{L, R}^{2}$. Specific uncertainties which may affect significantly neutrino cross sections are the relative size of up and down

of opposite-sign muons. Since they mainly proceed through scattering of the neutrino off a strange quark, they are a sensitive probe of the strange distribution. 
distributions at large $x$ [31, 32] and the size of the strange and charm component [33]. Both have been explored by MRST [23], which produce parton sets where all these features are varied in turn. Again, using these parton sets in turn, we find no significant variation of the predicted $R_{\nu}, R_{\bar{\nu}}$, and of the extracted $g_{L, R}^{2}$. If, on the contrary, we relax the assumption $s=\bar{s}$, which is implicit in all these parton sets, we find a shift of $R_{\mathrm{PW}}$ in good agreement with eq. (11). This conclusion appears to be robust, and only weakly affected by the choice of parton distributions and by the specific $x$-dependence of the $s-\bar{s}$ difference, provided the second moment of $s-\bar{s}$ is kept fixed. The lower cut $(20 \mathrm{GeV})$ imposed by $\mathrm{NuTeV}$ on the energy deposited in the calorimeter tends to decrease the sensitivity to the asymmetry $s^{-}$, as it mostly eliminates high $x$ events. However, this effect is relevant only for lower energy neutrinos, below about $100 \mathrm{GeV}$, and should be small in the case of $\mathrm{NuTeV}$.

The dependence on the choice of parton distributions is shown in fig. 1 where blue $\times($ red +$)$ crosses correspond to MRST99 (CTEQ) points. We cannot show a NuTeV value, because we could not access the parton set used by $\mathrm{NuTeV}$. The results are seen to spread along the expected PW line. The intercept of this line turns out to be determined by the input value of $g_{L}^{2}-g_{R}^{2}$, and to be completely insensitive to details of parton distributions. However, it should be kept in mind that inclusion of NLO corrections might alter significantly these results, by increasing the spread especially in the direction along the PW line, for the reasons discussed above.

Finally, we have tried to vary the charm mass, and to switch on some higher twist effects (specifically those related to the nucleon mass). In both cases the contributions to the uncertainty which we find are in agreement with those of $\mathrm{NuTeV}$.

\section{Oblique corrections}

After our review of the SM analysis, let us proceed with a discussion of possible effects of physics beyond the SM. We first concentrate on new physics which is characterized by a high mass scale and couples only or predominantly to the vector bosons. In this case its contributions can be parameterized in a model independent way by three (oblique) parameters. Among the several equivalent parameterizations [34], we adopt $\epsilon_{1}, \epsilon_{2}, \epsilon_{3}$ [35]. Many models of physics beyond the SM can be studied at least approximately in this simple way.

Generic contributions to $\epsilon_{1}, \epsilon_{2}, \epsilon_{3}$ shift $g_{L, R}^{2}$ according to the approximate expressions

$$
\frac{\delta g_{L}^{2}}{g_{L}^{2}}=2.8 \delta \epsilon_{1}-1.1 \delta \epsilon_{3} ; \quad \frac{\delta g_{R}^{2}}{g_{R}^{2}}=-0.9 \delta \epsilon_{1}+3.7 \delta \epsilon_{3} .
$$

Of course, the $\epsilon_{i}$ parameters are strongly constrained by electroweak precision tests. In order to see if this generic class of new physics can give rise to the NuTeV anomaly, we extract the $\epsilon_{i}$ parameters directly from a fit to the electroweak data, without using the SM predictions for them. We use the most recent set of electroweak observables, summarized in table 2, properly taking into account the uncertainties on $\alpha_{\mathrm{em}}\left(M_{Z}\right)$ and $\alpha_{\mathrm{s}}\left(M_{Z}\right)$. The result is a fit to the $\epsilon_{i}$ very close to the one reported in [20] which we use in eq. (12) after normalizing to the SM prediction at a reference value of $m_{h}$. The ellipses corresponding to the $68 \%$ and $99 \%$ CL are displayed in fig. 2 (green, almost horizontal ellipses). They are centred roughly around the SM best fit point, because the SM predictions for $\epsilon_{1}$ and $\epsilon_{3}$ for $m_{h} \approx 100 \mathrm{GeV}$ are in reasonable agreement with the data (see also section 2). The difference between the best fit point and the light Higgs SM prediction for $\left(g_{L}^{2}, g_{R}^{2}\right)$ is much smaller than the $\mathrm{NuTeV}$ accuracy. Notice that, as mentioned in section 2, excluding the hadronic asymmetries from the fit would make an oblique explanation even harder.

Our conclusion is that oblique corrections cannot account for the NuTeV anomaly, as they can only absorb about $\sim 1 \sigma$ of the $\sim 3 \sigma \mathrm{NuTeV}$ anomaly. 


$$
\begin{array}{rlll}
G_{\mathrm{F}} & =1.1663710^{-5} / \mathrm{GeV}^{2} & & \text { Fermi constant for } \mu \text { decay } \\
M_{Z} & =91.1875 \mathrm{GeV} & & \text { pole } Z \text { mass } \\
m_{t} & =(174.3 \pm 5.1) \mathrm{GeV} & & \text { pole top masss } \\
\alpha_{\mathrm{S}}\left(M_{Z}\right) & =0.119 \pm 0.003 & & \text { strong coupling } \\
\alpha_{\mathrm{em}}^{-1}\left(M_{Z}\right) & =128.936 \pm 0.046 & & \text { electromagnetic coupling } \\
M_{W} & =(80.451 \pm 0.033) \mathrm{GeV} & 1.8-\sigma & \text { pole } W \text { mass } \\
\Gamma_{Z} & =(2.4952 \pm 0.0023) \mathrm{GeV} & -0.4-\sigma & \text { total } Z \text { width } \\
\sigma_{h} & =(41.540 \pm 0.037) \mathrm{nb} & 1.6-\sigma & e \bar{e} \text { hadronic cross section at } Z \text { peak } \\
R_{\ell} & =20.767 \pm 0.025 & 1.1-\sigma & \Gamma(Z \rightarrow \text { hadrons }) / \Gamma\left(Z \rightarrow \mu^{+} \mu^{-}\right) \\
R_{b} & =0.21646 \pm 0.00065 & 1.1-\sigma & \Gamma(Z \rightarrow b \bar{b}) / \Gamma(Z \rightarrow \text { hadrons }) \\
R_{c} & =0.1719 \pm 0.0031 & -0.1-\sigma & \Gamma(Z \rightarrow c \bar{c}) / \Gamma(Z \rightarrow \text { hadrons }) \\
A_{L R}^{e} & =0.1513 \pm 0.0021 & 1.6-\sigma & \text { Left/Right asymmetry in } e \bar{e} \\
A_{L R}^{b} & =0.922 \pm 0.02 & -0.6-\sigma & \text { LR Forward/Backward asymmetry in } e \bar{e} \rightarrow b \bar{b} \\
A_{L R}^{c} & =0.670 \pm 0.026 & 0.1-\sigma & \text { LR FB asymmetry in } e \bar{e} \rightarrow c \bar{c} \\
A_{F B}^{\ell} & =0.01714 \pm 0.00095 & 0.8-\sigma & \text { Forward/Backward asymmetry in } e \bar{e} \rightarrow \ell \bar{\ell} \\
A_{F B}^{b}=0.099 \pm 0.0017 & -2.8-\sigma & \text { Forward/Backward asymmetry in } e \bar{e} \rightarrow b \bar{b} \\
A_{F B}^{c} & =0.0685 \pm 0.0034 & -1.7-\sigma & \text { Forward/Backward asymmetry in } e \bar{e} \rightarrow c \bar{c} \\
Q_{W} & =-72.5 \pm 0.7 & 0.6-\sigma & \text { atomic parity violation in Cs }
\end{array}
$$

Table 2: The electroweak data included in our fit [36]. The second column indicates the discrepancy with respect to the best SM fit.

\section{Corrections to gauge boson interactions}

We now discuss whether the $\mathrm{NuTeV}$ anomaly could be explained by modifying the couplings of the vector bosons. This possibility could work if new physics only affects the $\bar{\nu} Z \nu$ couplings, reducing the squared $\bar{\nu}_{\mu} Z \nu_{\mu}$ coupling by $(1.16 \pm 0.42) \%$ [1]. This shift is consistent with precision LEP data, that could not measure the $\bar{\nu} Z \nu$ couplings as accurately as other couplings (no knowledge of the LEP luminosity is needed to test charged lepton and quark couplings), and found a $Z \rightarrow \nu \bar{\nu}$ rate $(0.53 \pm 0.28) \%$ lower than the best-fit SM prediction. We could not construct a model that naturally realizes this intriguing possibility, because precision data test the $\bar{\mu} Z \mu$ and $\bar{\mu} W \nu_{\mu}$ couplings at the per mille accuracy. This generic problem is best understood by considering explicit examples. We first show that models where neutrinos mix with some extra fermion (thereby shifting not only the $\bar{\nu} Z \nu$ coupling, but also the $\bar{\ell} W \nu$ coupling) do not explain the $\mathrm{NuTeV}$ anomaly. Next, we discuss why a model where the $Z$ mixes with some extra vector boson (thereby shifting not only the $\bar{\nu} Z \nu$ coupling, but also the $Z$ couplings of other fermions) does not explain the NuTeV anomaly.

\section{Models that only affect the neutrino couplings}

This happens e.g. in models where the SM neutrinos mix with right-handed neutrinos (a 1\% mixing could be naturally obtained in extra dimensional models or in appropriate 4-dimensional models [37]). By integrating out the right-handed neutrinos, at tree level one obtains the low energy effective lagrangian

$$
\mathscr{L}_{\text {eff }}=\mathscr{L}_{\mathrm{SM}}+\epsilon_{i j} 2 \sqrt{2} G_{F}\left(H^{\dagger} \bar{L}_{i}\right) i \not \partial\left(H L_{j}\right),
$$


where $i, j=\{e, \mu, \tau\}, L_{i}$ are the lepton left-handed doublets, $H$ is the Higgs doublet, and $\epsilon_{i j}=$ $\epsilon_{j i}^{*}$ are dimensionless couplings. This peculiar dimension 6 operator only affects neutrinos. After electroweak symmetry breaking, it affects the kinetic term of the neutrinos, that can be recast in the standard form with a redefinition of the neutrino field. In this way, the $\bar{\nu}_{i} Z \nu_{i}$ and the $\bar{\nu}_{i} W \ell_{i}$ couplings become respectively $1-\epsilon_{i i}$ and $1-\epsilon_{i i} / 2$ lower than in the $\mathrm{SM}\left(\epsilon_{i i}\right.$ is positive: gauge couplings of neutrinos get reduced if neutrinos mix with neutral singlets). The $\mathrm{NuTeV}$ anomaly would require $\epsilon_{\mu \mu}=0.0116 \pm 0.0042$. However, a reduction of the $\bar{e} W \nu_{e}$ and $\bar{\mu} W \nu_{\mu}$ couplings increases the muon lifetime, that agrees at about the per-mille level with the SM prediction obtained from precision measurements of the electromagnetic coupling and of the $W$ and $Z$ masses. Assuming that no other new physics beyond the extra operator in eq. (13) is present, from a fit of the data in table 2 we find that a flavour-universal $\epsilon_{i i}$ is constrained to be $\epsilon_{i i}=(0 \pm 0.4) 10^{-3}$. This bound cannot be evaded with flavour non universal corrections, that are too strongly constrained by lepton universality tests in $\tau$ and $\pi$ decays [38]. In conclusion, $\epsilon_{\mu \mu}$ can possibly generate only a small fraction of the $\mathrm{NuTeV}$ anomaly.

In principle, the strong bound from muon decay could be circumvented by mixing the neutrinos with extra fermions that have the same $W$ coupling of neutrinos but a different $Z$ coupling. In practice, it is not easy to build such models.

\section{Models that only affect the $Z$ couplings}

Only the $Z$ couplings are modified, e.g., in models with an extra $\mathrm{U}(1) Z^{\prime}$ gauge boson that mixes with the $Z$ boson. The $Z^{\prime}$ effects can be described by the $Z Z^{\prime}$ mixing angle, $\theta$, by the $Z^{\prime}$ boson mass, $M_{Z^{\prime}}$, and by the $Z$ gauge current $J_{Z^{\prime}}$. At leading order in small $\theta$ and $M_{Z} / M_{Z^{\prime}}$, the tree-level low energy lagrangian gets modified in three ways.

(1) the SM prediction for the $Z$ mass gets replaced by $M_{Z}^{2}=M_{Z}^{2 \mathrm{SM}}-\theta^{2} M_{Z^{\prime}}^{2}$;

(2) the $Z$ current becomes $J_{Z}=J_{Z}^{\mathrm{SM}}-\theta J_{Z^{\prime}}$;

(3) at low energy, there are the four fermion operators generated by $Z^{\prime}$ exchange, beyond the ones generated by the $W_{ \pm}$and $Z$ bosons:

$$
\mathscr{L}_{\text {eff }}\left(E \ll M_{Z}, M_{Z^{\prime}}\right)=-\frac{J_{W_{+}} J_{W_{-}}}{M_{W}^{2}}-\frac{1}{2}\left[\frac{J_{Z}^{2}}{M_{Z}^{2}}+\frac{J_{Z^{\prime}}^{2}}{M_{Z^{\prime}}^{2}}\right]+\cdots .
$$

As discussed in section 1 , (1) cannot explain the $\sim 1 \% \mathrm{NuTeV}$ anomaly. Here we show that the same happens also for (2): the $Z$ couplings are constrained by LEP and SLD at the per-mille level, and less accurately by atomic parity violation data, as summarized in table 2. However, the less accurate of these data have $\sim 1 \%$ errors, and present some anomalies. The $Z \rightarrow \nu \bar{\nu}$ rate and the Forward/Backward asymmetries of the $b$ and $c$ quarks show a few- $\sigma$ discrepancy with the best-fit $\mathrm{SM}$ prediction. But the $Z \rightarrow b \bar{b}$ and $Z \rightarrow c \bar{c}$ branching ratios agree with the SM. The best SM fit, including also the $\mathrm{NuTeV}$ data [1], has $\chi^{2} \approx 30$ with 14 d.o.f. In this situation, it is interesting to study if these anomalies could have a common solution with $Z$ couplings about $1 \%$ different from the SM predictions. We therefore extract the $Z$ couplings directly from the data, without imposing the SM predictions for them. This kind of analysis has a general interest. Since we are here concerned with the $\mathrm{NuTeV}$ anomaly, we apply our results to compute the range of $\left(g_{L}^{2}, g_{R}^{2}\right)$ consistent with the electroweak data. We recall that both neutrino and quark couplings enter in determining $g_{L}^{2}$ and $g_{R}^{2}$.

We assume that the $Z$ couplings are generation universal and $\mathrm{SU}(2)_{L}$ invariant as in the SM: we therefore extract from the data the 5 parameters $g_{Q}, g_{U}, g_{D}, g_{L}$ and $g_{E}$ that describe the $Z$ 
couplings to the five kinds of SM fermions listed in table 1. In the context of $Z^{\prime}$ models, this amounts to assume that the $Z^{\prime}$ has generation-universal couplings that respect $\mathrm{SU}(2)_{L}$. This assumption of $\mathrm{SU}(2)_{L}$ invariance is theoretically well justified, although one could possibly invent some non minimal model where it does not hold. On the contrary, the universality assumption only has a pragmatic motivation: we cannot make a fit with more parameters than data.

We obtain the result shown by the large red ellipse on the right side of fig. 目. This generic class of models gives a best fit value close to the SM prediction. Although the error is much larger than in a pure SM fit, it does not allow to cleanly explain the $\mathrm{NuTeV}$ anomaly. We find that the global $\chi^{2}$ can be decreased by about 4 with respect to a SM fit: taking into account that we have five more parameters this is not a statistically significant reductionf in agreement with old similar analyses [39.

One could generalize this analysis in several directions. For example, new physics could shift the on-shell $Z$ couplings tested at LEP and SLD differently from the low-energy $Z$ couplings relevant for $\mathrm{NuTeV}$. Alternatively, there could be flavour dependent shifts of the $Z$ couplings. This happens e.g. in the model considered in [11, where it is suggested that the $\mathrm{NuTeV}$ anomaly could be reproduced by a mixing between the $Z$ boson with a $Z^{\prime}$ boson coupled to the lepton flavour numbers $L_{\mu}-L_{\tau}$. However, this mixing also shifts the couplings of charged $\tau$ and $\mu$ leptons, that are too precisely tested by LEP and SLD to allow for a significant fraction of the NuTeV anomaly.

\section{Loop effects in the MSSM}

It is well known that supersymmetric contributions to the electroweak precision observables decouple rapidly. Under the present experimental constraints it is very difficult to find regions of parameter space where radiative corrections can exceed a few per-mille. Explaining the NuTeV anomaly (a 1.2\% discrepancy with the SM prediction for $g_{L}^{2}$ ) with low-energy supersymmetry looks hopeless from the start. Moreover, the dominant contributions to $\epsilon_{1}$ in the MSSM are always positive [40]. It then follows from eqs. (12) that, in order to explain at least partially the measured value of $g_{L}^{2}$, the supersymmetric contributions to $\epsilon_{3}$ should be positive and of $\mathcal{O}(1 \%)$.

An interesting scenario which can be easily investigated is the one recently proposed in [20] to improve the global fit to the electroweak data. As the main contributions of squark loops would be a positive shift in $\epsilon_{1}$, all squarks can be assumed heavy, with masses of the order of one $\mathrm{TeV}$. Relatively large supersymmetric contributions are then provided by light gauginos and sleptons and can be parameterized in terms of only four supersymmetric parameters $(\tan \beta$, the Higgsino mass $\mu$, the weak gaugino mass $M_{2}$, and a supersymmetry-breaking mass of left-handed sleptons). The oblique approximation used in section 1 is not appropriate for light superpartners (sneutrinos can be as light as $50 \mathrm{GeV}$ ). We therefore consider the complete supersymmetric one-loop corrections in this scenario (see [20] and refs. therein). Taking into account the various experimental bounds on the chargino and slepton masses, we find the potential shifts to $g_{L, R}^{2}$ shown in fig. 3 . They are small and have the wrong sign. Low-energy supersymmetric loops cannot generate the NuTeV anomaly.

\footnotetext{
${ }^{4}$ On the contrary, if we allow for generation universal but non $\mathrm{SU}(2)_{L}$-invariant corrections of the $Z$ couplings to $u_{L}$ and $d_{L}$ and to $\nu$ and $e_{L}$, we get a statistically significant reduction in the global $\chi^{2}\left(\Delta \chi^{2} \approx 21\right.$ with 7 more parameters), due to the fact that various anomalies, including of course the $\mathrm{NuTeV}$ anomaly, can be explained in this artificial context. Without including the $\mathrm{NuTeV}$ data, the best fit regions in the $\left(g_{L}^{2}, g_{R}^{2}\right)$ plane are shifted towards the NuTeV region.
} 


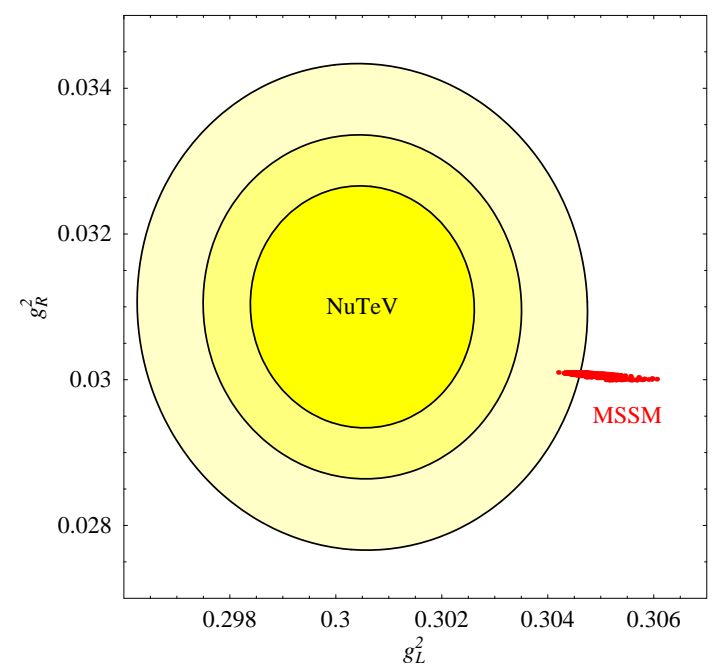

Figure 3: Shifts in $g_{L, R}^{2}$ in the supersymmetric scenario described in the text (light sleptons and gauginos) for points in the parameter space which are not excluded by experimental constraints.

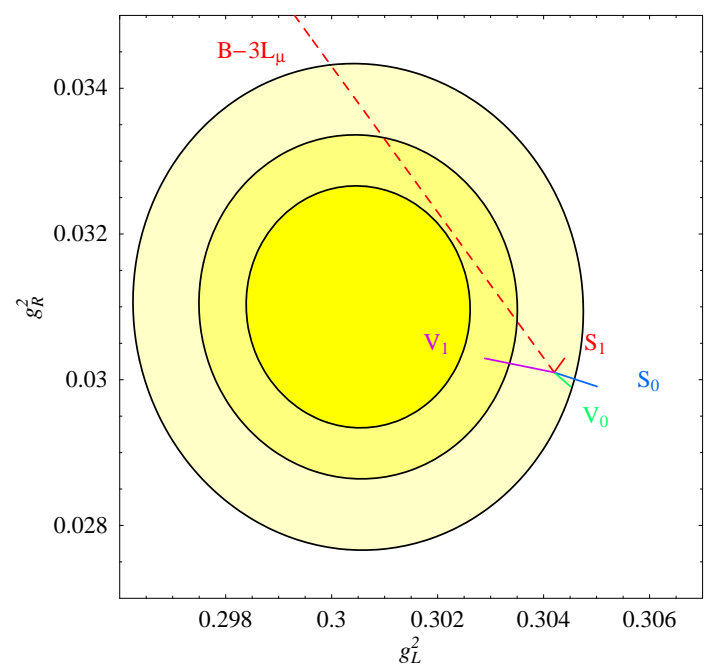

Figure 4: Deviations from the SM prediction that can be induced by minimal leptoquarks (continuous lines) and by an extra $B-3 L_{\mu}$ gauge boson (dashed red line) without conflicting by more than $1 \sigma$ with other bounds.

\section{Non renormalizable operators}

Non renormalizable operators parameterize the effects of any new physics too heavy to be directly produced. As discussed in sections 6 and 5, new physics that affects the $Z, W_{ \pm}$propagators or couplings cannot fit the NuTeV anomaly without some conflict with other electroweak data. We now consider dimension six lepton-lepton-quark-quark operators that conserve baryon and lepton number.

We start from a phenomenological perspective, with $\mathrm{SU}(3) \otimes \mathrm{U}(1)_{\mathrm{em}}$ invariant four fermion vertices, and determine which vertices could explain the $\mathrm{NuTeV}$ anomaly without conflicting with other data. Then we next consider which $\mathrm{SU}(2)_{L}$ invariant operators generate the desired four-fermion vertices. In the next sections, we will discuss new particles whose exchange could generate these operators.

Taking into account Fierz identities, the most generic Lagrangian that we have to consider can be written as

$$
\begin{aligned}
\mathscr{L}_{\mathrm{eff}}= & \mathscr{L}_{\mathrm{SM}}-2 \sqrt{2} G_{F}\left[\epsilon_{\bar{\ell}_{i} \ell_{j} \bar{q}_{r} q_{s}}^{A B}\left(\bar{\ell}_{i} \gamma^{\mu} P_{A} \ell_{j}\right)\left(\bar{q}_{r} \gamma_{\mu} P_{B} q_{s}\right)+\right. \\
& \left.+\delta_{\bar{\ell}_{i} \ell_{j} \bar{q}_{r} q_{s}}^{A B}\left(\bar{\ell}_{i} P_{A} \ell_{j}\right)\left(\bar{q}_{r} P_{B} q_{s}\right)+t_{\bar{i}_{i} \ell_{j} \bar{q}_{r} q_{s}}^{A A}\left(\bar{\ell}_{i} \gamma^{\mu \nu} P_{A} \ell_{j}\right)\left(\bar{q}_{r} \gamma_{\mu \nu} P_{A} q_{s}\right)\right] .
\end{aligned}
$$

where $\gamma_{\mu \nu}=\frac{i}{2}\left[\gamma_{\mu}, \gamma_{\nu}\right], P_{R, L} \equiv\left(1 \pm \gamma_{5}\right) / 2$ are the right- and left-handed projectors, $q$ and $\ell$ are any quark or lepton, $A, B=\{L, R\}$ and $\epsilon, \delta$ and $t$ are dimensionless coefficients. In order to explain the $\mathrm{NuTeV}$ anomaly, new physics should give a negative contribution to $g_{L}^{2}$. This can be accomplished by

1. reducing the NC $\nu_{\mu}$-nucleon cross section. The operators given by new physics must contain left-handed first generation quarks. 
2. increasing the $\mathrm{CC} \nu_{\mu}$-nucleon cross section. The quarks do not need to be left-handed, and the quark in the final state needs not to be of the first generation.

We now show that 'scalar' operators (the ones with coefficients $\delta$ ) cannot explain NuTeV, lefthanded 'vector' operators (with coefficient $\epsilon^{L L}$ ) can realize the first possibility and 'tensor' operators (with coefficient $t$ ) perhaps the second one.

The scalar operators with coefficient $\delta$ contribute to the charged current. In order to accommodate the $\mathrm{NuTeV}$ anomaly, these operators should appear with a relatively large coefficient $\delta \gtrsim 0.1$, since their contribution to CC scattering has only a negligible interference with the dominant SM amplitude. The interference is suppressed by fermion masses (for first generation quarks) or by CKM mixings (if a quark of higher generation is involved). For first generation quarks, this value of $\delta_{\bar{\mu} \nu_{\mu} \bar{u} d}$ is inconsistent with $R_{\pi}$. When new physics-SM interference is included in this ratio, it becomes 41]

$$
R_{\pi} \equiv \frac{\operatorname{BR}\left(\pi \rightarrow e \bar{\nu}_{e}\right)}{\operatorname{BR}\left(\pi \rightarrow \mu \bar{\nu}_{\mu}\right)}=R_{\pi}^{\mathrm{SM}}\left[1-2\left(\epsilon_{\bar{\mu} \nu_{\mu} \bar{u} d}^{L L}-\epsilon_{\bar{e} \nu_{e} \bar{u} d}^{L L}\right)-\frac{2 m_{\pi}^{2}}{m_{\mu}\left(m_{u}+m_{d}\right)} \delta_{\bar{\mu} \nu_{\mu} \bar{u} d}^{L P}\right] .
$$

The measured value, $R_{\pi}=(1.230 \pm 0.004) \times 10^{-4}$ [42], agrees with the SM prediction [43, 44]

$$
R_{\pi}^{\mathrm{SM}}=\frac{m_{e}^{2}\left(m_{\pi}^{2}-m_{e}^{2}\right)^{2}}{m_{\mu}^{2}\left(m_{\pi}^{2}-m_{\mu}^{2}\right)^{2}}\left(1-16.2 \frac{\alpha_{\mathrm{em}}}{\pi}\right)
$$

which implies $\delta_{\bar{\mu} \nu_{\mu} \bar{u} d} \lesssim 10^{-4}$. Furthermore, scalar operators which produce a $s, c$ or $b$ quark in the final state also cannot explain the $\mathrm{NuTeV}$ anomaly. The values of $\delta$ required would be in conflict with upper bounds on FCNC meson decays such as $K^{+} \rightarrow \pi^{+} \mu \bar{\mu}, D^{+} \rightarrow \pi^{+} \mu \bar{\mu}$, and $B^{0} \rightarrow \mu \bar{\mu}$.

Vector operators can possibly generate the $\mathrm{NuTeV}$ anomaly if they are of $L L$ type. Assuming first generation quarks, the operators in eq. (14) shift $g_{L}^{2}$ as

$$
\delta g_{L}^{2}=2\left[g_{L u} \epsilon_{\bar{\nu}_{\mu} \nu_{\mu} \bar{u} u}^{L L}+g_{L d} \epsilon_{\bar{\nu}_{\mu} \nu_{\mu} \bar{d} d}^{L L}-g_{L}^{2} \epsilon_{\bar{\nu}_{\mu} \mu \bar{d} u}^{L L}\right] .
$$

The CC term, $\epsilon_{\bar{\nu}_{\mu} \mu \bar{d} u}^{L L}$, cannot alone fit the NuTeV anomaly without overcontributing to the $\pi \rightarrow \mu \bar{\nu}_{\mu}$ decay. In principle, one could allow for cancellations between different contributions to $R_{\pi}$ in eq. (15). However LEP [45] (and bounds from atomic parity violation [36]) exclude the simplest possibility, $\epsilon_{\bar{\mu} \nu_{\mu} \bar{u} d}^{L L}=\epsilon_{\bar{e} \nu_{e} \bar{u} d}^{L L}$.

We now assume that these vector operators are generated by new physics heavier than the maximal energy of present colliders (about few hundred $\mathrm{GeV}$ ), and study the bounds from collider data. Operators involving second generation leptons are constrained by the Tevatron; LEP and HERA are not sensitive to them?. In the case of vector operators, the Tevatron sets a limit 46] $\left|\epsilon_{\bar{\mu} \mu \bar{q} q}^{L L}\right| \lesssim 0.03$ $(q=u$ is slightly more constrained than $q=d$, because protons contain more $u$ than $d$ ). Assuming $\mathrm{SU}(2)_{L}$-invariance (that relates the $\epsilon_{\bar{\ell} \ell \bar{q} q}^{L L}$ with $\ell=\left\{\mu, \nu_{\mu}\right\}$ and $q=\{u, d\}$, see below) the Tevatron bound is close but consistent with the value suggested by NuTeV, $\left|\epsilon_{\bar{\nu}_{\mu} \nu_{\mu} \bar{q} q}^{L L}\right| \sim 0.01$.

Tensor operators could possibly produce the $\mathrm{NuTeV}$ anomaly via mechanism 2 , because $\pi$-decays give no bound on $t_{\bar{\mu} \nu_{\mu} \bar{u} d}$ (using only the $\pi$ momentum it is not possible to write any antisymmetric tensor). Tensor operators have not been studied in [46], but if they are generated by physics at a scale

\footnotetext{
${ }^{5}$ Both at $\mathrm{NuTeV}$ and at CCFR these operators may induce deviations from the SM values of $g_{L}, g_{R}$. Within their errors, the CCFR data are consistent with both the SM and with NuTeV, as clearly shown in fig. 3 of [14]. Therefore CCFR bounds on non renormalizable operators, reported by the PDG [42], cannot conflict with $\mathrm{NuTeV}$.
} 
$\gg M_{Z}$, the value of $t_{\bar{\mu} \nu_{\mu} \bar{u} d} \sim 0.1$ necessary to fit the NuTeV anomaly is within (and probably above) the sensitivity of present Tevatron data. Furthermore we do not know how new physics (e.g. exchange of new scalar or vector particles [47]) could generate only tensor operators, without also generating the scalar operators that overcontribute to $R_{\pi}$. We will therefore focus on vector operators.

We now consider $\mathrm{SU}(2)_{L}$-invariant operators. We have shown that the $\mathrm{NuTeV}$ anomaly could be explained by the four-fermion vertex $\left(\bar{\nu}_{\mu} \gamma^{\mu} P_{L} \nu_{\mu}\right)\left(\bar{q}_{1} \gamma_{\mu} P_{L} q_{1}\right)$. Only two $\mathrm{SU}(2)_{L}$ invariant operators operators can generate this vertex

$$
\mathcal{O}_{L Q}=\left[\bar{L} \gamma_{\mu} L\right]\left[\bar{Q} \gamma_{\mu} Q\right], \quad \mathcal{O}_{L Q}^{\prime}=\left[\bar{L} \gamma_{\mu} \tau^{a} L\right]\left[\bar{Q} \gamma_{\mu} \tau^{a} Q\right]
$$

We left implicit the $\mathrm{SU}(2)_{L}$ indices, on which the Pauli matrices $\tau^{a}$ act. Other possible 4 fermion operators, with different contractions of the $\mathrm{SU}(2)_{L}$ indices, can be rewritten as linear combinations of these two operators.

The $\mathrm{NuTeV}$ anomaly can be fit by $\mathcal{O}_{L Q}$ if it is present in $\mathscr{L}_{\text {eff }}$ as $(-0.024 \pm 0.009) 2 \sqrt{2} G_{\mathrm{F}} \mathcal{O}_{L Q}$, as discussed above. The operator

$$
\mathcal{O}_{L Q}^{\prime}=\left[\bar{\nu}_{\mu} \gamma_{\mu} \nu_{\mu}-\bar{\mu}_{L} \gamma_{\mu} \mu_{L}\right]\left[\bar{u}_{L} \gamma_{\mu} u_{L}-\bar{d}_{L} \gamma_{\mu} d_{L}\right]+2\left[\bar{\mu}_{L} \gamma_{\mu} \nu_{\mu}\right]\left[\bar{u}_{L} \gamma_{\mu} d_{L}\right]+2\left[\bar{\nu}_{\mu} \gamma_{\mu} \mu_{L}\right]\left[\bar{d}_{L} \gamma_{\mu} u_{L}\right]
$$

also can fit the NuTeV anomaly. However its CC part overcontributes to $\pi \rightarrow \mu \bar{\nu}_{\mu}$, giving a contribution to $\epsilon_{\bar{\mu} \nu_{\mu} \bar{u} d}^{L L}$ about 10 times larger than what allowed by $R_{\pi}$, see eq. (15).

These operators could be induced e.g. by leptoquark or $Z^{\prime}$ boson exchange, which we study in the following two sections. A critical difference between these possibilities is that leptoquarks must be heavier than about $200 \mathrm{GeV}$ 48, 49, 50], whereas a neutral $Z^{\prime}$ boson could also be lighter than about $10 \mathrm{GeV}$ (see section 9). Leptoquarks are charged and coloured particles that would be pair-produced at colliders, if kinematically possible. If the $\mathrm{NuTeV}$ anomaly is due to leptoquarks, their effects should be seen at run II of the Tevatron or at the LHC. If instead the NuTeV anomaly were due to a weakly coupled light $Z^{\prime}$, it will not show up at Tevatron or LHC.

\section{Leptoquarks}

Leptoquarks are scalar or vector bosons with a coupling to leptons and quarks. In this section, we consider leptoquarks which induce baryon and lepton number conserving four-fermion vertices.

The symmetries of the SM allow different types of leptoquarks, which are listed in [51]. There are four leptoquarks that couple to $Q L$, so these are candidates to explain the $\mathrm{NuTeV}$ anomaly. They are the scalar $\mathrm{SU}(2)_{L}$ singlet $\left(S_{0}\right)$ and triplet $\left(S_{1}^{a}\right)$, and the vector $\mathrm{SU}(2)_{L}$ singlet $\left(V_{0 \mu}\right)$ and triplet $\left(V_{1 \mu}^{a}\right)$, with interaction Lagrangian

$$
\lambda_{S_{0}}[Q L] S_{0}+\lambda_{S_{1}}\left[Q \tau^{a} L\right] S_{1}^{a}+\lambda_{V_{0}}\left[\bar{Q} \gamma_{\mu} L\right] V_{0 \mu}+\lambda_{V_{1}}\left[\bar{Q} \gamma_{\mu} \tau^{a} L\right] V_{1 \mu}^{a}+\text { h.c. }
$$

We do not speculate on how the above leptoquarks could arise in specific models.

Consider first the scalar $S_{0}$. The lower bound on leptoquark masses from the Tevatron is $200 \mathrm{GeV} 48$, 49], therefore at NuTeV leptoquarks are equivalent to effective operators. Tree level exchange of $S_{0}$, with mass $m$ and coupling $\lambda\left[Q_{1} L_{2}\right] S_{0}$ (1 and 2 are generation indices), induces the four-fermion operator

$$
\mathscr{L}_{\mathrm{eff}}=\frac{|\lambda|^{2}}{m^{2}}\left(Q_{1} L_{2}\right)\left(Q_{1} L_{2}\right)^{\dagger}=\frac{|\lambda|^{2}}{4 m^{2}}\left(\mathcal{O}_{L Q}-\mathcal{O}_{L Q}^{\prime}\right),
$$




\begin{tabular}{cccccccc}
$\mathrm{LQ}$ & $\mathscr{L}_{\mathrm{eff}}$ & $\delta g_{L}^{2}$ & $\epsilon_{\bar{\nu}_{\mu} \nu_{\mu} \bar{d} d}^{L L}$ & $\epsilon_{\bar{\mu} \mu \bar{u} u}^{L L}$ & $\epsilon_{\bar{\nu}_{\mu} \nu_{\mu} \bar{u} u}^{L L}$ & $\epsilon_{\bar{\mu} \mu \bar{d} d}^{L L}$ & $\epsilon_{\bar{\nu}_{\mu} \mu \bar{d} u}^{L L}, \epsilon_{\bar{\mu} \nu_{\mu} \bar{u} d}^{L L}$ \\
\hline \hline$S_{0}$ & $\frac{|\lambda|^{2}}{4 m^{2}}\left(\mathcal{O}_{L Q}-\mathcal{O}_{L Q}^{\prime}\right)$ & $0.12 \alpha$ & $-\alpha / 2$ & $-\alpha / 2$ & 0 & 0 & $\alpha / 2$ \\
\hline \multirow{2}{*}{$S_{1}$} & $\frac{|\lambda|^{2}}{4 m^{2}}\left(\mathcal{O}_{L Q}^{\prime}+3 \mathcal{O}_{L Q}\right)$ & $0.03 \alpha$ & $-\alpha / 2$ & $-\alpha / 2$ & 0 & 0 & $-\alpha / 2$ \\
\hline$V_{0}$ & $-\frac{|\lambda|^{2}}{2 m^{2}}\left(\mathcal{O}_{L Q}^{\prime}+\mathcal{O}_{L Q}\right)$ & $0.09 \alpha$ & 0 & 0 & $\alpha$ & $\alpha$ & $\alpha$ \\
\hline & & & 0 & $2 \alpha$ & 0 & 0 & 0 \\
$V_{1}$ & $\frac{|\lambda|^{2}}{2 m^{2}}\left(\mathcal{O}_{L Q}^{\prime}-3 \mathcal{O}_{L Q}\right)$ & $-0.40 \alpha$ & 0 & 0 & $\alpha$ & $\alpha$ & $-\alpha$ \\
& & & 0 & 0 & 0 & 0 & 0
\end{tabular}

Table 3: The four leptoquarks that generate the operators suggested by the NuTeV anomaly. Columns two and three are the effective Lagrangian and the contribution to $g_{L}^{2}$ (in terms of $\alpha \equiv\left|\lambda^{2}\right| / 2 \sqrt{2} G_{\mathrm{F}} m^{2}$ ) for leptoquarks with $\mathrm{SU}(2)_{L}$ degenerate masses. In the last columns, we give the coefficients of the individual four fermion operators, separating the triplet members.

The sign of the operator is fixed, because the coupling constant is squared. We see that $S_{0}$ cannot explain the $\mathrm{NuTeV}$ anomaly, because it generates $\mathcal{O}_{L Q}$ with the wrong sign (it gives a positive contribution to $g_{L}^{2}$ ), and because it also generates the unwanted operator $\mathcal{O}_{L Q}^{\prime}$.

In the context of supersymmetric models without $R$-parity $S_{0}$ can be identified with a $\tilde{D}_{g}^{c}$ squark of generation $g$ and superpotential interaction $\lambda_{2 g 1}^{\prime} L_{2} D_{g}^{c} Q_{1}$. It is interesting to explore further the possible contributions of $R$-parity violating squarks at NuTeV. In supersymmetric models, $\tilde{D}^{c}$ is accompanied by a scalar $\mathrm{SU}(2)_{L}$ doublet squark (leptoquark), $\tilde{Q}$. The exchange of $\tilde{Q}$ only modifies the right-handed coupling $g_{R}$, so that it cannot explain $\mathrm{NuTeV}$ by itself. Mixing of right- and lefthanded squarks generates dimension seven operators. This mixing is usually, but not always, negligibly small (e.g. one can consider large $\tan \beta$, or non minimal models). The relevant $\Delta L=2$ four-fermion operators are

$$
\lambda_{i j k}^{\prime} \lambda_{m n j}^{\prime}\left[\bar{d}_{k} P_{L} \nu_{i}\right]\left[\bar{Q}_{n}^{c} P_{L} L_{m}\right]=\lambda_{i j k}^{\prime} \lambda_{m n j}^{\prime}\left[\bar{d}_{k} P_{L} \nu_{i}\right]\left[\bar{u}_{n}^{c} P_{L} e_{m}-\bar{d}_{n}^{c} P_{L} \nu_{m}\right]
$$

These operators cannot account for the NuTeV anomaly: they do not interfere with the SM amplitude and contribute to both $\mathrm{NC}$ and $\mathrm{CC}$, leading to a positive correction to $g_{L}$.

In table 3, we list the effective four-fermion operators, and the contribution to $g_{L}^{2}$, of $S_{0}, S_{1}, V_{0}$ and $V_{1}$. In the $\mathscr{L}_{\text {eff }}$ column we have assumed that the members of triplet leptoquarks are degenerate. Only the vector $\mathrm{SU}(2)_{L}$ triplet leptoquark gives a negative contribution to $g_{L}^{2}$. In all cases $\mathcal{O}_{L Q}$ is generated together with the unwanted $\mathcal{O}_{L Q}^{\prime}$ operator, that overcontributes to the $\pi \rightarrow \mu \bar{\nu}_{\mu}$ decay, as discussed in the previous section. These features are also shown in fig. 自, where we plot the deviations from the SM prediction induced by the $S_{0}, S_{1}, V_{0}, V_{1}$ leptoquarks imposing that they should not overcontribute to $R_{\pi}$ by more than $1 \sigma$.

In the subsequent columns of table 3 we generalize the effective Lagrangian assuming that $\mathrm{SU}(2)_{L}$ breaking effects split the triplets in a general way. In this situation, the scalar and vector $\mathrm{SU}(2)_{L}$ triplet leptoquarks can explain the $\mathrm{NuTeV}$ anomaly. In the scalar (vector) case, $\mathrm{NuTeV}$ can be fit by 
reducing the mass of the triplet member that induces $\epsilon_{\bar{\nu}_{2} \nu_{2} \bar{u} u}^{L L}\left(\epsilon_{\bar{\nu}_{2} \nu_{2} \bar{d} d}^{L L}\right)$, by a factor of $\sqrt{2}$. From [52], we expect that such split multiplets are consistent with precision electroweak measurements.

We conclude that the $\mathrm{NuTeV}$ anomaly cannot be generated by $\mathrm{SU}(2)_{L}$ singlet or doublet leptoquarks, or by triplet leptoquarks with degenerate masses. However, triplet leptoquarks with carefully chosen mass splittings between the triplet members can fit the $\mathrm{NuTeV}$ data - and this explanation should be tested at Run II of the Tevatron or at LHC.

\section{$9 \quad$ Unmixed extra $\mathrm{U}(1) Z^{\prime}$ boson}

The sign of the dimension 6 lepton/quark operators generated by an extra $Z^{\prime}$ vector boson depends on the lepton and quark charges under the $\mathrm{U}(1)^{\prime}$ gauge symmetry. Therefore, with generic charges, it is possible to generate a correction to neutrino/nucleon scattering with the sign suggested by the $\mathrm{NuTeV}$ anomaly. In order to focus on theoretically appealing $Z^{\prime}$ bosons, we require that

- Quark and lepton mass terms are neutral under the extra $\mathrm{U}(1)^{\prime}$. We make this assumption because experimental bounds on flavour and CP-violating processes suggest that we do not have a flavour symmetry at the electroweak scale.

- The $Z^{\prime}$ couples to leptons of only second generation. Bounds from (mainly) LEP2 445] and older $e \bar{e}$ colliders would prevent to explain the $\mathrm{NuTeV}$ anomaly in presence of couplings to first generation leptons. We avoid couplings to third generation leptons just for simplicity.

- The extra $\mathrm{U}(1)^{\prime}$ does not have anomalies that require extra light fermions charged under the SM gauge group.

The only gauge symmetry that satisfies these conditions is $B-3 L_{\mu}$ (for related work see [53]), where $B$ is the baryon number and $L_{\mu}$ is the muon number. If Under these restrictions, the sign of the $Z^{\prime}$ correction to neutrino/nucleon scattering is fixed, and this $Z^{\prime}$ allows to fit the NuTeV anomaly. In fact, the four-fermions operators generated by $Z^{\prime}$ exchange are

$$
\mathscr{L}_{Z^{\prime}}=-\frac{g_{Z^{\prime}}^{2}}{2\left(M_{Z^{\prime}}^{2}-t\right)}\left[\bar{Q} \gamma_{\mu} Q-\bar{U}^{c} \gamma_{\mu} U^{c}-\bar{D}^{c} \gamma_{\mu} D^{c}-9 \bar{L}_{2} \gamma_{\mu} L_{2}+9 \bar{E}_{\mu}^{c} \gamma_{\mu} E_{\mu}^{c}\right]^{2}
$$

where $t$ is momentum transferred: $t \sim-20 \mathrm{GeV}^{2}$ at $\mathrm{NuTeV}$. The best fit of the NuTeV anomaly is obtained for (see fig. 田)

$$
\sqrt{M_{Z^{\prime}}^{2}-t} \approx g_{Z^{\prime}} 3 \mathrm{TeV}
$$

We now discuss the experimental bounds on such a $Z^{\prime}$.

\section{Collider bounds}

The bounds from Tevatron 56

$$
\sigma\left(p \bar{p} \rightarrow Z^{\prime} X \text { at } \sqrt{s}=1.8 \mathrm{TeV}\right) \operatorname{BR}\left(Z^{\prime} \rightarrow \mu \bar{\mu}\right)<40 \mathrm{fb} \quad(95 \% \mathrm{CL})
$$

\footnotetext{
${ }^{6}$ The neutrino masses suggested by oscillation data 54 do not respect $L_{\mu}$. If we allow third generation leptons to be charged under the extra $\mathrm{U}(1)^{\prime}$ symmetry, we could have a $B-c L_{\mu}-(3-c) L_{\tau}$ gauge group ( $c$ is a constant). However even a $L_{\mu} \pm L_{\tau}$ symmetry would not force a successful pattern of neutrino masses and mixings, that rather suggest a flavour symmetry containing $L_{e}-L_{\mu}-L_{\tau}$ [55].
} 
and LEP [57]

$$
\operatorname{BR}\left(Z \rightarrow \mu \bar{\mu} Z^{\prime}\right) \operatorname{BR}\left(Z^{\prime} \rightarrow \mu \bar{\mu}\right) \lesssim \text { few } \times 10^{-6}
$$

imply that $M_{Z^{\prime}}$ cannot be comparable to $M_{Z}$. One needs either a light $Z^{\prime}, M_{Z^{\prime}} \lesssim 10 \mathrm{GeV}$, or a heavy $Z^{\prime}, M_{Z^{\prime}} \gtrsim 600 \mathrm{GeV}$ [56]. Perturbativity implies $M_{Z^{\prime}} \lesssim 5 \mathrm{TeV}$.

\section{The anomalous magnetic moment of the muon}

The $Z^{\prime}$ gives a correction to the anomalous magnetic moment of the muon. Assuming $M_{Z^{\prime}} \gg m_{\mu}$, we get

$$
a_{\mu}=a_{\mu}^{\mathrm{SM}}+\frac{27 g_{Z^{\prime}}^{2}}{4 \pi^{2}} \frac{m_{\mu}^{2}}{M_{Z^{\prime}}^{2}}=a_{\mu}^{\mathrm{SM}}+8.410^{-10}\left(\frac{3 \mathrm{TeV}}{M_{Z^{\prime}} / g_{Z^{\prime}}}\right)^{2}
$$

At the moment, the $a_{\mu}$ measured by the $g-2$ collaboration [4] is slightly higher than the SM prediction, $a_{\mu}^{\exp }-a_{\mu}^{\mathrm{SM}}=(20 \pm 18) 10^{-10}$, if one employs $a_{\mu}^{\text {had }}=(697 \pm 10) 10^{-10}$ for the hadronic polarization contribution [6] and $a_{\mu}^{\mathrm{lbl}}=(9 \pm 2) 1^{-10}$ for the light-by-light contribution [5]. One could still prefer to estimate it as $a_{\mu}^{\mathrm{lbl}}=(0 \pm 10) 10^{-10}$, obtaining a larger discrepancy and error.

Therefore, if $M_{Z^{\prime}} / g_{Z^{\prime}} \approx 3 \mathrm{TeV}$, as suggested by the NuTeV anomaly in the heavy $Z^{\prime}$ case, the $Z^{\prime}$ correction to the $g-2$ is comparable to the sensitivity of present experiments. If instead $M_{Z^{\prime}} \lesssim 5 \mathrm{GeV}$, the $Z^{\prime}$ that fits the $\mathrm{NuTeV}$ anomaly gives a larger correction to the muon $g-2$, see eq. (19). For example, for $M_{Z^{\prime}} \approx 3 \mathrm{GeV}$ one can fit the central value of $a_{\mu}^{\exp }-a_{\mu}^{\mathrm{SM}}$. On the other hand, a $Z^{\prime}$ lighter than $(1 \div 2) \mathrm{GeV}$ cannot explain the $\mathrm{NuTeV}$ anomaly without overcontributing to $a_{\mu}$. Similar light $Z^{\prime}$ models were proposed [58] as a possible source of the discrepancy between $a_{\mu}^{\exp }$ and previous SM computations 助.

\section{Other bounds}

Quantum corrections generate an unwanted kinetic mixing between the $Z^{\prime}$ and the SM hypercharge boson [59]. A light $Z^{\prime}$ needs a small gauge coupling $g_{Z^{\prime}}$, making these quantum effects negligible.

The $Z^{\prime}$ could contribute to the decay of $q \bar{q}$ mesons into $\bar{\mu} \mu$. This is negligibly small for $g_{Z^{\prime}} \sim$ $M_{Z^{\prime}} / 3 \mathrm{TeV}$, unless $m_{Z^{\prime}}$ is very close to the meson mass $m_{q \bar{q}}$. There are various $c \bar{c}$ mesons in the few $\mathrm{GeV}$ mass range, but $\Gamma_{Z^{\prime}}$ is very narrow, so the $Z^{\prime}$ is only ruled out in narrow windows $m_{Z^{\prime}} \approx$ $m_{q \bar{q}} \pm \Gamma_{q \bar{q}}[6]$.

The $Z^{\prime}$ that can fit the $\mathrm{NuTeV}$ anomaly does not give significant corrections to rare $K, D$ and $B$ decays. Let us consider for example the $K^{+} \rightarrow \pi^{+} \nu \bar{\nu}$ decay. This is a sensitive probe because the dominant $\mathrm{SM} Z$ penguins that know that $\mathrm{SU}(2)_{L}$ is broken (and can therefore generate the FCNC vertex $m_{t}^{2}\left[\bar{s}_{L} \gamma_{\mu} Z_{\mu} d_{L}\right]$, with GIM cancellations spoiled by the large top mass) are suppressed by the small mixing $V_{t s}^{*} V_{t d}$. On the contrary, penguin loops of quarks that do not know that $\mathrm{U}(1)^{\prime}$ is broken (that therefore only generate the $q^{2}\left[\bar{s}_{L} \gamma_{\mu} Z_{\mu}^{\prime} d_{L}\right]$ operator, where $q$ is the $Z^{\prime}$ momentum and the GIM cancellations is only spoiled by logarithms of quark masses) are suppressed only by the larger Cabibbo mixing $V_{c d}$. The $Z^{\prime}$ suggested by $\mathrm{NuTeV}$ gives a negligible correction even to this favorable $K^{+} \rightarrow \pi^{+} \nu \bar{\nu}$ decay.

\footnotetext{
${ }^{7}$ The total number of measured $Z \rightarrow \mu \bar{\mu} \mu \bar{\mu}$ agrees with the SM prediction and there is no peak in the $\mu \bar{\mu}$ invariant mass. In order to extract a precise bound on the $Z^{\prime}$ mass from these data one should take into account the experimental cuts and resolution.

${ }^{8} \mathrm{~A} Z^{\prime}$ boson with mass $M_{Z^{\prime}} \sim 100 \mathrm{MeV}$ (this case is not motivated by NuTeV) would mediate the resonant decay $K^{+} \rightarrow \pi^{+} Z^{\prime} \rightarrow \pi^{+} \nu \bar{\nu}$, producing an excess of monoenergetic $\pi^{+}$in the $K^{+}$rest frame, compatibly with the first experimental data [61].
} 
Ref. [9] claims a statistically significant hint of dimuon events (three events, versus an estimated background of $0.07 \pm 0.01$ events), possibly generated by some neutral long lived particle with production cross section $\sim 10^{-10} / \mathrm{GeV}^{2}$ of few $\mathrm{GeV}$ mass (see also [62]). The $Z^{\prime}$ suggested by $\mathrm{NuTeV}$ can have the right mass and cross section, but is not sufficiently long lived. However, it could partially decay in sufficiently long lived neutral fermions. Extra light neutral fermions are required to cancel gravitational anomalies and $\mathrm{U}(1)^{\prime 3}$ anomalies.

\section{$Z^{\prime}$ burst and the GZK cutoff}

The $Z^{\prime}$ gives a narrow resonant contribution to $\nu \bar{\nu}$ scattering, which could perhaps generate ultrahigh energy cosmic ray events with $E \sim 10^{20} \mathrm{GeV}$. The analogous $Z$ resonance [63] has been considered as a possible source of the observed events above the Greisen-Zatsepin-Kuzmin (GZK) cutoff [64]. A cosmic ray neutrino that scatters with a nonrelativistic cosmic microwave background neutrino would encounter the $Z^{\prime}$ resonance at the energy

$$
E_{\nu}^{Z^{\prime} \text { res }}=\frac{M_{Z^{\prime}}^{2}}{2 m_{\nu}}=10^{20} \mathrm{eV}\left(\frac{M_{Z^{\prime}}}{3 \mathrm{GeV}}\right)^{2} \frac{0.05 \mathrm{eV}}{m_{\nu}}
$$

where we have used a neutrino mass suggested by atmospheric oscillation data [54. A resonance at a $Z^{\prime}$ mass of few $\mathrm{GeV}$ is more suitable than a resonance on the $Z$, where a larger incident neutrino energy $E_{\nu}^{Z \text { res }}=M_{Z}^{2} / 2 m_{\nu} \gtrsim 4 \times 10^{21} \mathrm{eV}$ would be required, even if neutrinos are as heavy as possible, $m_{\nu} \lesssim 1 \mathrm{eV}$. The $Z$ burst scenario is problematic because it seems difficult to imagine a cosmological source that produces enough very energetic neutrinos without producing, at the same time, too many photons.

Although the $Z^{\prime}$ requires less energetic cosmic neutrinos than the $Z$, roughly the same total flux 63 is required in the two cases, because the $Z$ and the $Z^{\prime}$ have comparable energy-averaged cross sections. In fact the $\mathrm{NuTeV}$ and $g-2$ data suggest $\Gamma_{Z^{\prime}} / M_{Z^{\prime}} \sim \Gamma_{Z} / M_{Z}$ and

$$
\sigma\left(\nu \bar{\nu} \rightarrow Z^{\prime} \rightarrow f\right) \approx \frac{12 \pi^{2} \Gamma_{Z^{\prime}}}{M_{Z^{\prime}}} \mathrm{BR}\left(Z^{\prime} \rightarrow f\right) \mathrm{BR}\left(Z^{\prime} \rightarrow \nu \bar{\nu}\right) \delta\left(s-M_{Z^{\prime}}^{2}\right)
$$

where $f$ is any final state. In conclusion, a $Z^{\prime}$ burst could generate the observed cosmic rays above the GZK cutoff more easily than the $Z$ burst.

\section{Summary}

We have studied the possible origin of the $\mathrm{NuTeV}$ anomaly. Our main results are:

- QCD effects. Because of the approximate use of the Paschos-Wolfenstein relation, the discrepancy between the $\mathrm{NuTeV}$ result and the SM prediction is fairly independent of which set of standard parton distribution functions is employed. This is no longer true if one drops some of the simplifying assumptions which are usually made in the PDF fits. A small asymmetry between the momentum carried by strange and antistrange quarks in the nucleon, suggested by a recent analysis of neutrino data [10], could explain half of the discrepancy between $\mathrm{NuTeV}$ and the SM. Such an asymmetry has been set to zero in more recent global parton sets, but the value suggested in [10] seems compatible with the data used in these fits. It would be desirable to update the analysis of [10] including more recent di-muon data from $\mathrm{NuTeV}$ [28], though settling the issue is likely to require more detailed information, such as it could be achieved at a neutrino factory [65]. A tiny violation of isospin symmetry of parton distributions, largely 
compatible with current data, would have a similar impact. Both these effects may have to be taken into account in the evaluation of the systematic error.

- Generic corrections to the propagators or couplings of the SM gauge bosons can only produce a small fraction of the $\mathrm{NuTeV}$ anomaly, as shown in fig. 2. In order to perform such a general analysis we have extracted the 'oblique' parameters and the SM gauge couplings directly from a fit of precision data, without imposing the SM predictions. We have assumed that the $Z$ couplings are generation universal and respect $\mathrm{SU}(2)_{L}$, as in the SM. In principle, the $\mathrm{NuTeV}$ anomaly could be explained by new physics that only shifts the $\bar{\nu} Z \nu$ couplings. However this situation is not realized by mixing the $Z$ with extra vector bosons, nor by mixing the neutrino with extra fermions.

- MSSM. Loop corrections in the MSSM have generally the wrong sign and are far too small to contribute significantly to the $\mathrm{NuTeV}$ observables.

- Contact operators. Dimension six quark-quark-lepton-lepton operators can fit the $\mathrm{NuTeV}$ anomaly consistently with other data. The desired operators are neutral current, left-handed four fermion vertices of the form $\eta\left(\bar{\nu}_{\mu} \gamma^{\alpha} \nu_{\mu}\right)\left(\bar{q} \gamma_{\alpha} P_{L} q\right)$, where $q=\{u, d\}$. The coefficient $\eta$ must be of order $0.01 \times 2 \sqrt{2} G_{F}$, and the sign is fixed by requiring a negative interference with the SM. Effects of these operators should be seen at run II of Tevatron, unless they are generated by very weakly coupled light particles. If one restricts the analysis to $\mathrm{SU}(2)_{L}$-invariant operators, only the operator $\left[\bar{Q}_{1} \gamma^{\alpha} Q_{1}\right]\left[\bar{L}_{2} \gamma_{\alpha} L_{2}\right]$ can fit $\mathrm{NuTeV}$.

- Leptoquarks. SU(2) $L$ singlet and triplet leptoquarks can induce these operators — but if the leptoquark masses are $\mathrm{SU}(2)_{L}$ degenerate, either the sign is wrong or other unacceptable operators are also generated. A SU $(2)_{L}$ triplet leptoquark of spin one is a partial exception (see fig. (4) at least from a purely phenomenological perspective. Non degenerate triplet leptoquarks could fit the $\mathrm{NuTeV}$ results, but squarks in $R$-parity violating supersymmetry cannot.

- Extra U(1) vector bosons. A $Z^{\prime}$ boson that does not mix with the $Z$ boson can generate the NuTeV anomaly (see fig. 团), if its gauge group is $B-3 L_{\mu}$, the minimal choice suggested by theoretical and experimental inputs. The $Z^{\prime}$ can be either heavy, $600 \mathrm{GeV} \lesssim M_{Z^{\prime}} \lesssim 5 \mathrm{TeV}$, or light, $1 \mathrm{GeV} \lesssim M_{Z^{\prime}} \lesssim 10 \mathrm{GeV}$. The $Z^{\prime}$ that fits the NuTeV anomaly also increases the muon $g-2$ by $\sim 10^{-9}$ and (if light) gives a $Z^{\prime}$ burst to cosmic rays just above the GZK cutoff without requiring neutrino masses heavier that what suggested by oscillation data.

Acknowledgments We thank E. Barone, G. Giudice, G. Isidori, M. Mangano, K. McFarland, R.G. Roberts, A. Rossi and I. Tkachev for useful discussions. A.S. thanks R. Rattazzi for his insight into ionization corrections to the $\mu^{+}$range in iron. This work is partially supported by Spanish MCyT grants PB98-0693 and FPA2001-3031, by the Generalitat Valenciana under grant GV99-3-101, by the TMR network contract HPRN-CT-2000-00148 of the European Union, and by EU TMR contract FMRX-CT98-0194 (DG 12-MIHT). S.D. is supported by the Spanish Ministry of Education in the program "Estancias de Doctores y Tecnólogos Extranjeros en España" and P.G. by a EU Marie Curie Fellowship.

Note added The $\nu_{\mu}\left(\bar{\nu}_{\mu}\right) \mathrm{NuTeV}$ beam contains a 1.7\% contamination of $\nu_{e}\left(\bar{\nu}_{e}\right)$. A recent paper 66] suggests an explanation of the $\mathrm{NuTeV}$ anomaly assuming that $20 \%$ of $\nu_{e}, \bar{\nu}_{e}$ oscillate into sterile neutrinos. The suggested oscillation parameters are not consistent with disappearance experiments 
(unless Bugey and Chooz underestimated the theoretical error on their reactor fluxes). Furthermore, $\mathrm{NuTeV}$ not only predicts the $\nu_{e}$ and $\bar{\nu}_{e}$ fluxes through a MonteCarlo simulation, but also measures them directly (see pag. 26 of the transparencies in [1]). The agreement between the two determinations, at the few \% level, contradicts the oscillation interpretation.

Note added after publication (June 2002) In a recent publication 67, the NuTeV collaboration investigated the effect of a strange quark asymmetry and of isospin violation on their electroweak result. Specifically, they claim that the strange quark asymmetry is severely constrained by the dimuon data of Ref. [28], and that the effect of strange quark asymmetry and isospin violation might be considerably diluted due to the fact that $\mathrm{NuTeV}$ does not measure directly the Paschos-Wolfenstein ratio $R_{\mathrm{PW}}$, Eq. (6).

The claim that the dimuon (i.e. $\nu s \rightarrow \mu c$ scattering) data [28, 67] provides evidence against the strange asymmetry $s^{-} \approx+210^{-3}$ of the BPZ global fit [10] (and in fact suggest a negative asymmetry $s^{-}=-(2.7 \pm 1.3) 10^{-3}$ [28, 67]) appears dubious, for the following reasons:

1. The parametrization of the strange and antistrange distributions assumed by $\mathrm{NuTeV}$ is unphysical, in that it violates the constraint that the proton and neutron carry no strangeness. Furthermore, due to its too small number of free parameters, it artificially relates the $s / \bar{s}$ asymmetry at $x<0.5$ (where $\mathrm{NuTeV}$ have significant data) to the one at $x>0.5$ (where $\mathrm{NuTeV}$ have few events).

2. NuTeV did not make a global fit allowing a strange asymmetry, but rather made a fit to their dimuon data, using a set of parton distributions based on pre-existing (now obsolete) fits, obtained neglecting NLO QCD corrections and optimized under the assumption $s=\bar{s}$. This is especially worrisome due to the fact that the strange asymmetry found by $\mathrm{NuTeV}$ appears to depend very sensitively on the underlying set of parton distributions (see table I of [28]).

3. The dimuon data are considerably less sensitive to $\bar{s}$ than to $s$. In fact, the claim [28, 67] that a strange asymmetry at $x>0.5$ is excluded at high confidence level should be restated as the statement that $\mathrm{NuTeV}$ rules out a total strangeness at $x>0.5$ of the magnitude found by BPZ [10]. However, what matters for the $\mathrm{NuTeV}$ anomaly is the strange asymmetry.

The BPZ global fit [10] is not subject to these drawbacks, but it did not include the recent dimuon data. The BPZ fit is characterized by a relatively large strange sea at large $x$, driven mainly by CDHSW data, which agrees well with positivity constraints derived from polarized DIS [68]. Hence, the only conclusion that can be drawn by comparing these two analyses is that the size of the large- $x$ strange sea suggested by CDHSW as analyzed by BPZ seems larger than allowed by NuTeV data [28]. The origin of this discrepancy, and its impact on the best-fit strange asymmetry, could only be assessed by performing a global NLO fit which includes all available data. Our statement in section 3 remains therefore unchanged: the impact of the data [28] on the strange asymmetry is unclear.

$\mathrm{NuTeV}$ also comment on isospin violating parton distributions [67], taking the model by Thomas et al. 69] as reference. This model predicts a small effect on $s_{\mathrm{W}}^{2}$, as a result of a subtle cancellation between high and low $x$ regions. This conclusion is model-dependent. The fact remains that $\mathcal{O}(1 \%)$ isospin violation effects could generate the $\mathrm{NuTeV}$ anomaly, without conflicting with any other existing data.

Coming to the possible dilution of strangeness asymmetry or isospin violation due to experimental cuts, we should like to point out that, contrary to what stated in [67], we did include charm threshold effects and some experimental cuts in our analysis. We found moderate dilution effects, as discussed 
in section 3 of this paper. On the other hand, we cannot simulate the full NuTeV experimental set-up. However, if what $\mathrm{NuTeV}$ really measures differs from $R_{\mathrm{PW}}$ in a way which is significant at the required level of accuracy, then the cancellation of NLO effects that occurs in the Paschos-Wolfenstein relation cannot be taken for granted any longer. In particular, NuTeV claims to be less sensitive to $R_{\bar{\nu}}$ than is $R_{\mathrm{PW}}$. In general any asymmetry between charged-current and neutral-current, or between $\nu$ and $\bar{\nu}$ events spoils the cancellation of the NLO corrections in Eq. (11). Such asymmetries can also be induced by experimental cuts and by different $\nu, \bar{\nu}$ spectra. If any of these effects were significant, a $\mathrm{NLO}$ analysis would be required in order to obtain a reliable determination of $s_{\mathrm{W}}^{2}$ at the desired level of accuracy. Only a full NLO analysis of the $\mathrm{NuTeV}$ data could settle this issue.

\section{References}

[1] The NuTeV collaboration, hep-ex/011005; K. McFarland, seminar available at the internet address www.pas.rochester.edu/ ${ }^{\sim} \mathrm{ksmcf} / \mathrm{NuTeV} /$ seminaronly-fnaloct26.pdf

[2] CERN NA31 collaboration, Phys. Lett. B317 (1993) 233; Fermilab KTev collaboration, Nucl. Phys. Proc. Suppl. 96 (2001) 301; CERN NA48 collaboration, hepex/0110019

[3] S. Bertolini et al., Nucl. Phys. B514 (1998) 93 (hepph/9706260); A. Buras et al., Nucl. Phys. B592 (2001) 55; E. Pallante, A. Pich, I. Scimemi, Nucl. Phys. B617 (2001) 441, and refs. therein.

[4] The muon $g-2$ collaboration, Phys. Rev. Lett. 86 (2001) 2227 (hep-ex/0102017).

[5] M. Knecht, A. Nyffeler, hep-ph/011105s; M. Hayakawa, T. Kinoshita, hep-ph/011210\%; E. Bartos et al., hep-ph/0106084; I. Blokland, A. Czarnecki, K. Melnikov, hep-ph/0112117; J. Bijnens, E. Pallante, J. Prades, hep-ph/011225s.

[6] F. Jegerlehner, hep-ph/0105283; see also M. Davier, A. Hocker, Phys. Lett. B419 (1998) 419. For reviews see e.g. W.J. Marciano, B.L. Roberts, hep-ph/0105056 and K. Melnikov, Int. J. Mod. Phys. A16 (2001) 4591 hep-ph/010526\%).

[7] C. S. Wood, S. C. Bennett, D. Cho, B. P. Masterson, J. L. Roberts, C. E. Tanner and C. E. Wieman, Science 275 (1997) 1759.

[8] A. Derevianko, Phys. Rev. Lett. 85 (2000) 1618; M.G. Kozlov, S.G. Porsev, I. I. Tupitsyn, Phys. Rev. Lett. 86 (2001) 3260; V.A. Dzuba, C. Harabati, W.R. Johnson, M.S. Safronova, Phys. Rev. A63 (2001) 44103. A.I. Milstein, O. P. Sushkov, hep-ph/010925

[9] T. Adams et al., Phys. Rev. Lett. 87 (2001) 41801 hep-ex/0104037.

[10] V. Barone, C. Pascaud, F. Zomer, Eur. Phys. J. C12 (2000) 243 hep-ph/9907512.

[11] E. Ma, D.P. Roy, hep-ph/0111385

[12] E.A. Paschos, L. Wolfenstein, Phys. Rev. D7 (1973) 91.

[13] K. S. McFarland et al., NuTeV collaboration, hepex/9806013.
[14] K. S. McFarland et al., CCFR collaboration, Eur. Phys. J. C1 (1998) 509 hep-ex/9701010.

[15] A. Sirlin, Phys. Rev. D22 (1980) 971.

[16] W. J. Marciano, A. Sirlin, Phys. Rev. D22 (1980) 2695 erratum ibid. D31, 213 (1980).

[17] P. Gambino, hep-ph/9812339 and refs. therein.

[18] G. Degrassi, P. Gambino, Nucl. Phys. B567 (2000) 3.

[19] M. Grünewald, private communication. See also 36.

[20] G. Altarelli et al., JHEP 106 (2001) 18 hepph/0106029).

[21] M.S. Chanowitz, hep-ph/0104024.

[22] W. Furmanski, R. Petronzio, Z. Phys. bf C11 (1982) 293.

[23] A. D. Martin, R. G. Roberts, W. J. Stirling and R. S. Thorne, Eur. Phys. J. C4 (1998) 463; Eur. Phys. J. C 14 (2000) 133.

[24] S. Forte, Phys. Rev D47 (1993) 1842; F. G. Cao and A. I. Signal, Phys. Rev. C62 (2000) 015203.

[25] S. J. Brodsky, P. Hoyer, C. Peterson and N. Sakai, Phys. Lett. B93 (1980) 451.

[26] S.J. Brodsky, B.Q. Ma, Phys. Lett. B381 (1996) 317 hep-ph/9604393);

[27] CCFR collaboration, A.O. Bazarko et al., Z. Phys. C65 (1995) 189 hep-ex/9406007.

[28] The NuTeV collaboration, M. Goncharov et al., Phys. Rev. D64 (2001) 112006.

[29] R. G. Roberts private communication

[30] CTEQ collaboration, H.L. Lai et al., Eur. Phys. J. C12 (2000) 375 hep-ph/9903289.

[31] U.K. Yang, A. Bodek, Phys. Rev. Lett. 82 (1999) 2467 hep-ph/980948().

[32] S. Kuhlmann et al., Phys. Lett. B476 (2000) 291 hepph/9912283).

[33] S. Kretzer et al., Phys. Rev. D64 (2001) 033003 hepph/0101088.

[34] M. E. Peskin and T. Takeuchi, Phys. Rev. Lett. 65 (1990) 964; D. C. Kennedy, P. Langacker, Phys. Rev. Lett. 65 (1990) 2967 and erratum ibid. 66 (1990) 2967. 
[35] G. Altarelli, R. Barbieri, Phys. Lett. B253 (1991) 161.

[36] The LEP electroweak working group, see http://www.web.cern.ch/LEPEWWG.

[37] See e.g. J. Bernabéu et al., Phys. Lett. B187 (1987) 303; A. de Gouvea et al., hep-ph/010715 and references therein.

[38] For a review see e.g. A. Pich, Nucl. Phys. Proc. Suppl. 98 (2001) 385 (hep-ph/001229 $).$

[39] For a relatively recent analysis, performed when atomic parity violation seemed to contain an anomaly, see J. Erler and P. Langacker, Phys. Rev. Lett. 84 (2000) 212.

[40] M. Drees and K. Hagiwara, Phys. Rev. D42 (1990) 1709.

[41] O. Shanker, Nucl. Phys. B204 (1982) 375; O. Shanker, Nucl. Phys. B206 (1982) 253; S. Davidson, D.C. Bailey, B.A. Campbell, Z. Phys. C 61 (1994) 613 hepph/9309310.

[42] D.E. Groom et al., The European Physics Journal C15 (2000) 1.

[43] W. J. Marciano and A. Sirlin, Phys. Rev. Lett. 71 (1993) 3629; D.I. Britton et al., Phys. Rev. Lett. 68 (1992) 3000; G. Czapek et al., Phys. Rev. Lett. 70 (1993) 17

[44] M. Finkemeier, Phys. Lett. B387 (1996) 391 hepph/9505434).

[45] The LEP and SLD collaborations, hep-ex/0103049.

[46] The CDF collaboration, Phys. Rev. Lett. 79 (1997) 2198.

[47] A. Pich and J. P. Silva, Phys. Rev. D52 (1995) 4006 hep-ph/950532y.

[48] The D0 collaboration, Phys. Rev. Lett. 84 (2000) 2088 hep-ex/9910040.

[49] The CDF collaboration, Phys. Rev. Lett. 81 (1998) 4806 hep-ex/9810024).

[50] The OPAL collaboration, hep-ex/0112024.

[51] W. Buchmuller, R. Ruckl, D. Wyler, Phys. Lett. B191 (1987) 442 and erratum ibid. B448 (1987) 320.
[52] See e.g. S. Bergmann, Y. Grossman, D.M. Pierce, hepph/9909390

[53] L.N. Chang, O. Lebedev, W. Loinaz, T. Takeuchi, Phys. Rev. D63 (2001) 74013 hep-ph/0010118.

[54] The SuperKamiokande collaboration, Phys. Rev. Lett. 85 (2000) 3999 (hep-ex/000900); The MACRO collaboration, Phys. Lett. B517 (2001) 59 hep$e x / 0106049$.

[55] R. Barbieri et al., Phys. Lett. B445 (1999) 407 hepph/9808333).

[56] The CDF collaboration, Phys. Rev. Lett. 79 (1997) 2192.

[57] ALEPH collaboration, Z. Phys. C66 (1995) 3; See also E. Ma, D.P. Roy, S. Roy, hep-ph/011014

[58] S.N. Gninenko, N.V. Krasnikov, Phys. Lett. B513 (2001) 119 hep-ph/0102222.

[59] B. Holdom, Phys. Lett. B166 (1986) 196.

[60] D.C. Bailey and S. Davidson, Phys. Lett. B348 (1995) 185 hep-ph/9411355 C.D. Carone and H. Murayama, Phys. Rev. Lett. 74 (1995) 3122 hep-ph/9411256.

[61] E787 collaboration, hep-ex/0111091.

[62] C. Liu, Mod. Phys. Lett. A16 (1699) 2001; A. Dedes, H. Dreiner, P. Richardson, Phys. Rev. D65 (015001) 2002.

[63] T. Weiler, Phys. Rev. Lett. 49 (1982) 234 and erratum ibidem 12 (2000) 379; E. Roulet, Phys. Rev. D47 (1993) 5247; O.E. Kalashev et al., hep-ph/0112351. For a recent review see e.g. A. Ringwald, hep$p h / 0111112$ and ref.s therein.

[64] K. Greisen, Phys. Rev. Lett. 16 (1966) 748.

[65] M.L. Mangano et al., hep-ph/010515s.

[66] C. Giunti, M. Laveder, hep-ph/0202152

[67] NuTeV collaboration, hep-ex/0203004.

[68] S. Forte, M. L. Mangano, G. Ridolfi, Nucl. Phys. B602 (2001) 585 hep-ph/0101192).

[69] E.N. Rodionov, A.W. Thomas, J.T. Londergan, Mod. Phys. Lett. A9 (1994) 1799. 